\title{
Equatorial Deep Jets and Abyssal Mixing in the Indian Ocean
}

\author{
MARCUS DENGLER \\ Institut für Meereskunde an der Universität Kiel, Kiel, Germany \\ DETLEF QuADFASEL \\ Niels Bohr Institutet for Astronomi, Fysik og Geofysik, Københavns Universitet, Copenhagen, Denmark
}

(Manuscript received 13 January 1999, in final form 3 August 2001)

\begin{abstract}
Vertical profiles of horizontal currents and hydrographic measurements from three cruises along $80.5^{\circ} \mathrm{E}$ from the coast of Sri Lanka to $6^{\circ} \mathrm{S}$ between December 1990 and September 1994 are used to investigate the scales of the Indian Ocean deep jets as well as internal wave parameters and dissipation at the equator. The deep jets at $80.5^{\circ} \mathrm{E}$ have a vertical wavelength of $660 \mathrm{sm}$ (stretched meters) and amplitudes exceeding $10 \mathrm{~cm} \mathrm{~s}^{-1}$ in zonal velocity. They are observed throughout the water column and their flow direction reverses at $2^{\circ}$ off the equator. The vertical positions of the jets differ among the cruises and are consistent with a flow reversal between the data collected in winter and summer. During September 1994, the jets were less pronounced. Due to the meridional distribution of their zonal velocity and the phase relationship between zonal velocity and vertical displacement, the jets are best described as nondispersive first-mode equatorial Rossby waves. The hydrographic data revealed thick layers of low stratification with vertical scales of $15-55 \mathrm{~m}$ in the upper $2000 \mathrm{~m}$ of the water column. They are found primarily within $1^{\circ}$ of the equator and there is some evidence of correlation between the vertical position as well as the extent and the high strain zones of the deep jets. At vertical wavenumbers larger than those of the deep jets, shear and strain levels are five times larger than at off-equatorial locations and the compliant internal wave range ("roll-off range") begins at a smaller wavenumber ( $\left.k_{c} \approx 0.02 \mathrm{cpsm}\right)$. An estimate of the average dissipation rate within the deep jets yielded $\bar{\epsilon}=7.5 \times 10^{-10} \mathrm{~W} \mathrm{~kg}^{-1}$ between 500 - and 2000$\mathrm{m}$ depth. The elevated finescale internal wave field appears to be the main cause for the existence of the low stratification layers.
\end{abstract}

\section{Introduction}

Deep zonal equatorial currents with short vertical scales throughout a substantial part of the water column were discovered by Luyten and Swallow (1976) in the Indian Ocean. By now, the equatorial deep jets are known to exist in all oceans. In general, they are characterized by alternating east-west currents trapped within $1^{\circ}$ of the equator, having amplitudes of $10-20 \mathrm{~cm}$ $\mathrm{s}^{-1}$ and vertical wavelengths between 300 and $700 \mathrm{~m}$.

As more direct velocity measurements of the jets become available, temporal variability of the features as well as differences in vertical scale in three oceans have become evident. Observations from the western Indian Ocean collected during June 1976 showed an energetic maximum at $720 \mathrm{sm}$ (stretched meters) wavelength and a weaker but statistically significant maximum centered at $360 \mathrm{sm}$ in zonal velocity autospectra (O'Neill and Luyten 1984). The jets persisted over the 1-month-long

Corresponding author address: Dr. Marcus Dengler, FB1: Ozeanzirkulation und Klima (PO 1), Institut für Meereskunde, Düsternbrooker Weg 20, 24105 Kiel, Germany.

E-mail: mdengler@ifm.uni-kiel.de observation period and were coherent over $2.5^{\circ}$ of longitude. A phase reversal between $1^{\circ} 30^{\prime} \mathrm{N}$ and $3^{\circ} \mathrm{N}$ was significant. In contrast, Ponte and Luyten (1990) did not find statistically significant maxima in zonal wavenumber spectra in profiles of horizontal velocity from the western Indian Ocean. Their data were collected during April and June 1979 and showed only slightly enhanced energy in zonal velocity at 500 and $429 \mathrm{sm}$ wavelength.

In the Atlantic, deep jets were first inferred from hydrographic data by Eriksen (1982). Direct measurements by Ponte et al. (1990) showed alternating eastward and westward flows between 300 and $2200 \mathrm{~m}$. The meridional velocity showed similar oscillatory patterns with depth, but no apparent correlation to the zonal component was found. A station originally occupied by Ponte et al. (1990) in January 1989 was revisited in June 1991 (Böning and Schott 1993). Although a similar vertical structure was found, the currents were of opposite direction below 700-m depth. A recent investigation in the central Atlantic by Gouriou et al. (1999) suggests seasonal reversal of the jets (though their data were collected $2.5 \mathrm{yr}$ apart). They report a dominant wave- 
length for the jets in the upper $2500 \mathrm{~m}$ of the water column of 400 and $600 \mathrm{~m}$. Results from an eddy-resolving, primitive equation model simulation forced with seasonal varying winds for the Atlantic Ocean also produced baroclinic long equatorial Rossby waves at annual period (Böning and Schott 1993), but the vertical scale of the model currents was somewhat larger than what is observed (Gouriou et al. 1999).

For the Pacific, Ponte and Luyten (1989) reported equatorial deep jet vertical wavelength centered at 350 $\mathrm{sm}$, with jets significantly correlated over $10^{\circ}$ of longitude. Similar results were obtained by Muench et al. (1994), who determined a vertical wavelength of 330 $\pm 70 \mathrm{~m}$. Furthermore, they showed the flow to reverse at $2^{\circ}$ off the equator at the scales of the deep jets. Over a period of 16 months the central Pacific jets were nearly stationary at the equator, showing little or no vertical phase propagation (Firing 1987).

Three ideas have been forwarded to explain the short vertical-scale structure of the equatorial deep jets. Several authors employed a concept of forced equatorial waves. Wunsch (1977) modeled a vertically structured equatorial flow field forced at the surface with the seasonal monsoon. However, the meridional and vertical structure of the model flow field had larger scales than suggested by the observations (O'Neill and Luyten 1984; Muench et al. 1994). Moreover, vertical propagation of the deep jets, which is a characteristic in Wunsch's model, has not been observed (Firing 1987; Ponte and Luyten 1989; O'Neill and Luyten 1984).

Hua et al. (1997) proposed an alternative jet mechanism where near-zero Ertel potential vorticity values allow for transfer of angular momentum between the two hemispheres and inertial deep jets are a byproduct of angular momentum mixing. Although this theory successfully accounts for the cores of the jets, which are displaced to the north of the equator, the reversal of the flow at higher latitudes is not reproduced. Also, the model studies have so far not included the observed temporal variability of the deep jets.

Most commonly, the model of free linear equatorial waves is used to rationalize deep jets (e.g., O'Neill and Luyten 1984; Ponte and Luyten 1989; Muench et al. 1994). Muench et al. (1994) showed that the meridional distribution of zonal velocity and the potential vorticity structure of the Pacific deep jets is consistent with a first meridional mode nondispersive Rossby wave. Ponte (1989) as well as Kawase (1987) modeled free equatorial waves with short vertical scale jets using Kelvin waves generated by deep western boundary currents and reflecting at the eastern boundary into long Rossby waves. However, the solutions are vertically propagating. Additionally, Wang et al. (1994) showed that Kawase's solutions cannot produce as substantial equatorial currents within the appropriate range of meridional scales as indicated by the observation.

Although the generation mechanism of the deep jets remains unclear, ideas exist on how a stacked jet struc- ture at the equator can be sustained despite kinetic energy loss due to dissipation or reflection at meridional boundaries. Muench and Kunze (1999) and Muench and Kunze (2000) showed that an energy transfer between the internal wave field and the deep jets occurs through momentum flux divergence and that internal waves encountering vertical critical layers can substantially accelerate the deep jets.

There are few and conflicting statements about how dissipation in the abyssal equatorial ocean is associated with the deep jets. Moum et al. (1986) report weaker microstructure activity on the equator below 300-m depth as compared to off-equatorial locations in the $\mathrm{Pa}$ cific, whereas Gregg (1976) finds no latitudinal differences. More recently, Gregg et al. (1995) supported Gregg's (1976) previous results, showing that although shear and strain are more energetic at the equator, as also reported by Toole and Hayes (1984), there is no enhancement of average dissipation. However, dissipation is modulated within the deep jets themselves with high values occurring in the shear zones (Gregg 1998).

Here we present observations from the central equatorial Indian Ocean at $80.5^{\circ} \mathrm{E}$ that document energetic structures of deep currents with short vertical scales. Four meridional sections of hydrographic data and three full depth sections of horizontal velocity profiles collected on three cruises in different seasons over a time span of nearly four years are used to determine the scales of the equatorial deep currents and to describe their temporal behavior. Our study finds the deep jets to have the meridional structure of equatorial Rossby waves. The hydrographic data show large step structures in temperature and salinity in the vicinity of the equator. To quantify their characteristics, shear and strain spectral levels are calculated. Last, the turbulence parameterization of Kunze et al. (1990) is used to estimate dissipation rates at the equator.

\section{Data}

Hydrographic and current profile measurements along $80.5^{\circ} \mathrm{E}$ (WOCE section IR8) from the coast of Sri Lanka across the equator to at least $6^{\circ} \mathrm{S}$ were carried out during three cruises between December 1990 and September 1994. In addition, two arrays of current meter moorings (WOCE ICM8) were deployed for periods of a year each (Schott et al. 1994; Reppin et al. 1999). The aim of the program was to study the seasonal variability of the monsoon current and its interaction with the equatorial flow field.

The data presented here is a subset of the above observations and consists of conductivity-temperaturedepth (CTD) profile measurements and vertical profiles of horizontal velocity recorded with an acoustically tracked Pegasus dropsonde (Fig. 1). From December 1990 to January 1991, R/V Sonne sailed from Sri Lanka southward to $6^{\circ} \mathrm{S}$ where it turned around and sailed back to Sri Lanka, thereby completing two sections on $80.5^{\circ} \mathrm{E}$ 


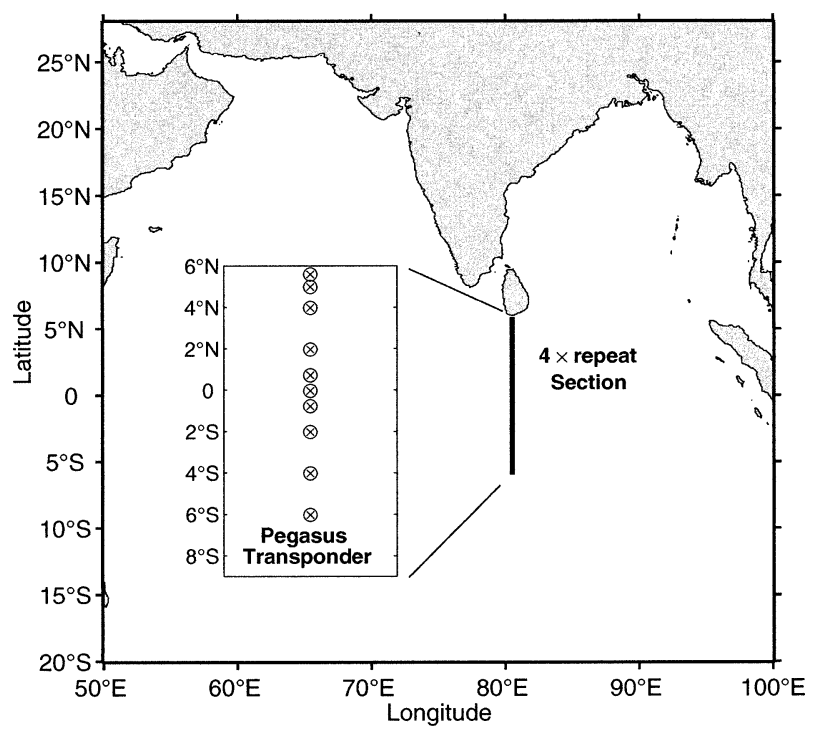

FIG. 1. Location of the study area in the Indian Ocean. The subplot shows the position of the Pegasus transponder $(\otimes)$ used on three cruises.

during cruise SO73. Another section was completed by R/V Sonne cruise SO88 in July 1993 and a fourth occupation was made by R/V Franklin cruise FR8/94 in September 1994. A total of 120 CTD profiles and 83 current profiles are available from these sections along $80^{\circ} \mathrm{E}$ between $6^{\circ} \mathrm{S}$ and the continental shelf of Sri Lanka at $6^{\circ} \mathrm{N}$.

The accuracy of CTD data meets the WOCE data quality standard for precision and accuracy of WHP (Joyce and Corry 1991). For CTD measurements a Neil Brown Mark III or a Seabird SBE911 was used. After calibration the data from R/V Sonne were interpolated onto a 1 decibar $(\mathrm{db})$ regular grid, while a vertical spacing of $2 \mathrm{db}$ was used for the R/V Franklin data. The overall error of absolute measurement was less than 2 $\times 10^{-3} \mathrm{~K}$ for temperature and less than $2 \times 10^{-3}$ for salinity (PSS-78). The absolute error of the pressure values was less than $3 \mathrm{db}$. About $40 \%$ of the collected CTD profiles were taken down to the bottom while for reasons of time saving the remaining casts were terminated at depths between 1500 and $2000 \mathrm{~m}$.

Ten pairs of transponder stations (Fig. 1) were deployed on R/V Sonne cruise SO73 for Pegasus measurements. Pegasus is a free-falling profiler that measures horizontal velocity (Spain et al. 1981). To improve the accuracy of the reference frame, real-time navigation was used for positioning the transponders (Send et al.

FIG. 2. Vertical profiles of horizontal current collected during SO73, SO88, and FR94 on $80.5^{\circ} \mathrm{E}$. The coherent current fluctuations with small vertical scales found within $1^{\circ}$ of the equator are shaded. (top) Eastward (U) and (bottom) northward (V) currents are gray; westward and southward currents are black.
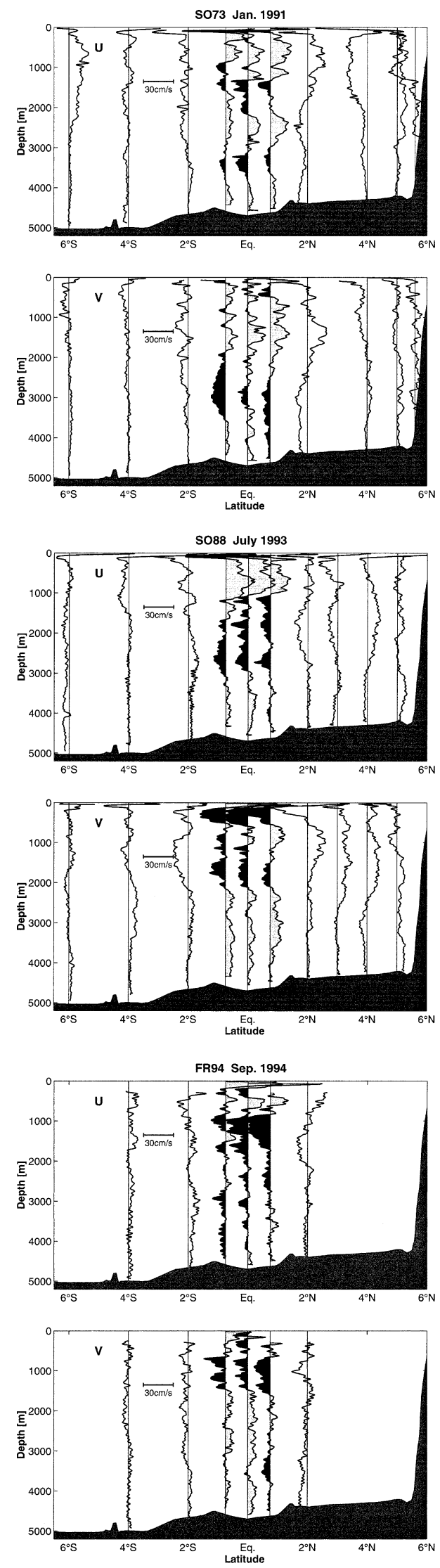


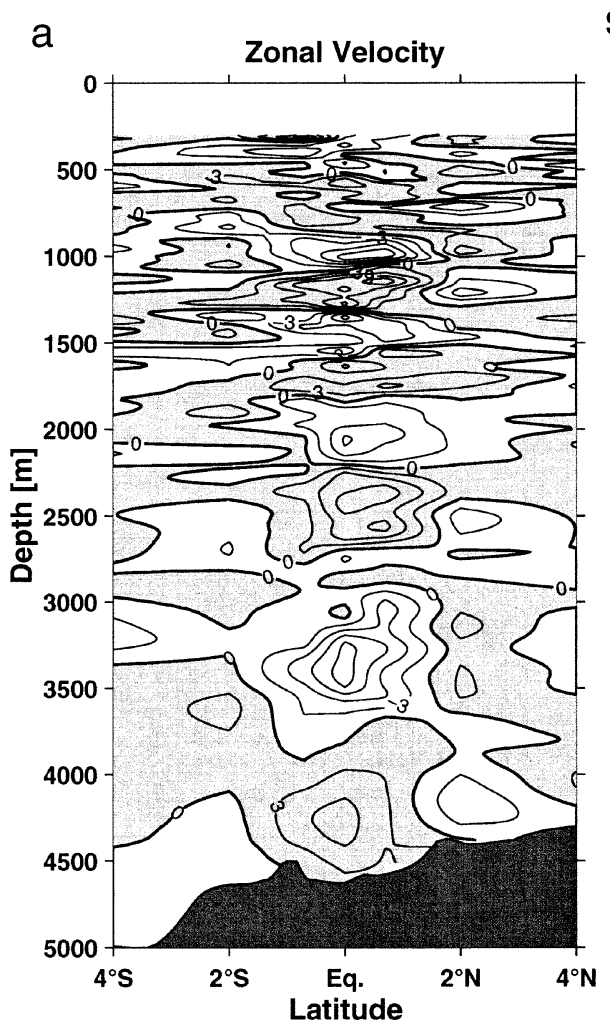

SO73

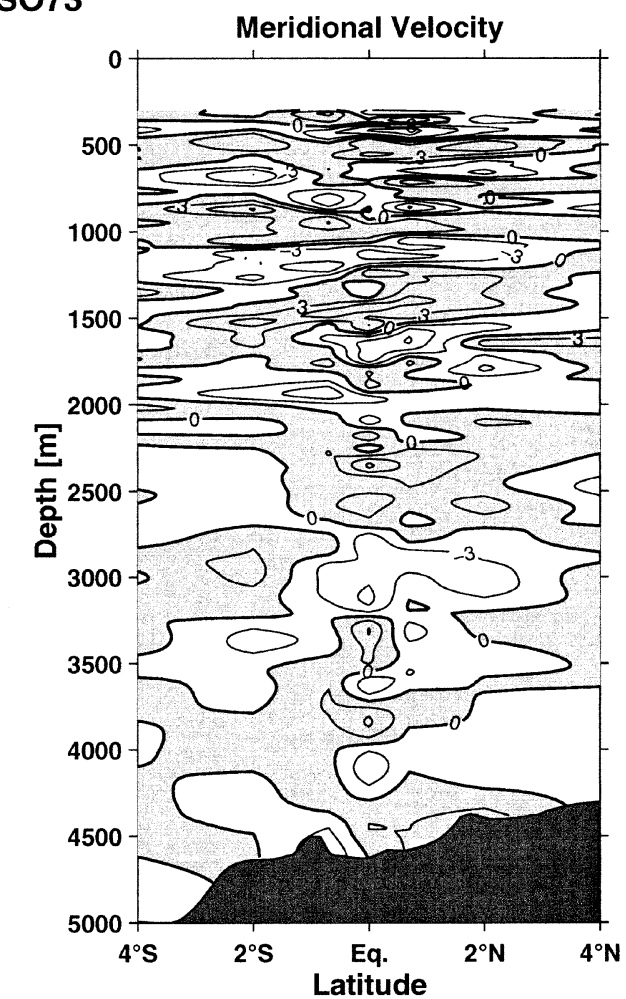

FIG 3. Meridional structure of the high vertical mode (left) zonal and (right) meridional velocity field (modes $>10$ ) along $80.5^{\circ} \mathrm{E}$ during (a) SO73, (b) SO88, and (c) FR94. Contour intervals are $3 \mathrm{~cm} \mathrm{~s}^{-1}$. Eastward and northward currents are gray.

1996). The Pegasus dropsonde was adjusted to have different descent and ascent velocities, 60 and $45 \mathrm{~cm}$ $\mathrm{s}^{-1}$, respectively, throughout all cruises. The vertical sampling interval obtained was $9.6 \mathrm{~m}$ for downcasts and $7.2 \mathrm{~m}$ for upcasts. Four sets (up and downcast) of current profiles are available from most transponder locations, resulting in a total of 83 current profiles. Of these, 58 reached to the bottom, while the maximum depth of the other casts was about $1500 \mathrm{~m}$, resulting in three full depth sections of horizontal velocity. Raw velocity profiles were low-pass filtered with a 50-m cutoff wavelength to remove small-scale instrumental noise and interpolated onto a 5-m vertical grid. The absolute error in the velocity after filtering is less than $2 \mathrm{~cm} \mathrm{~s}^{-1}$ (Send 1994; Send et al. 1996). Transponder power was running low in September 1994 when R/V Franklin occupied the section. Energetic small-scale fluctuations due to noise with vertical wavelengths of up to $100 \mathrm{~m}$ overlaid the current structure in some profiles. Measurement error of these profiles is somewhat larger than for those collected on the R/V Sonne cruises.

The CTD and current profiles were collected simultaneously. The dropsonde was deployed between 5 and 15 min prior to the start of the CTD cast and surfaced not later than $30 \mathrm{~min}$ after the end of the CTD cast. Horizontal displacement between the two measurements did not exceed $1.5 \mathrm{~km}$.

\section{Observations}

\section{a. Equatorial currents}

The zonal and meridional velocity profiles are presented in Fig. 2. The deep circulation within $1^{\circ}$ of the equator (shaded profiles) shows pronounced coherent currents alternating with depth, having vertical wavelengths between 200 and $1000 \mathrm{~m}$. Peak velocities exceed $\pm 20 \mathrm{~cm} \mathrm{~s}^{-1}$ below 300-m depth. In contrast to previously reported observations in the Indian Ocean (Luyten and Swallow 1976; Ponte and Luyten 1990) such high speeds are seen in both velocity components, although amplitudes at vertical wavelengths between 300 and 800 $\mathrm{m}$ are larger in zonal velocity. The flow field away from the equator is dominated by lower vertical modes, except close to the continental slope of Sri Lanka, where an increase in energy at small vertical scales is apparent.

Within $1^{\circ}$ of the equator at a depth of $50-150-\mathrm{m}$ westward flow with velocities of up to $40 \mathrm{~cm} \mathrm{~s}^{-1}$ was found in January 1991 (SO73) and July 1993 (SO88). In September 1994 (FR94) the flow there was eastward with a maximum velocity above $70 \mathrm{~cm} \mathrm{~s}^{-1}$. This strong current is the Indian Ocean Equatorial Undercurrent, which is generally present only during the transition phase between the monsoons in March-May but was also observed in August-September, 1994 (Reppin et al. 1999). The depth range 300-900 $\mathrm{m}$ had predomi- 
b

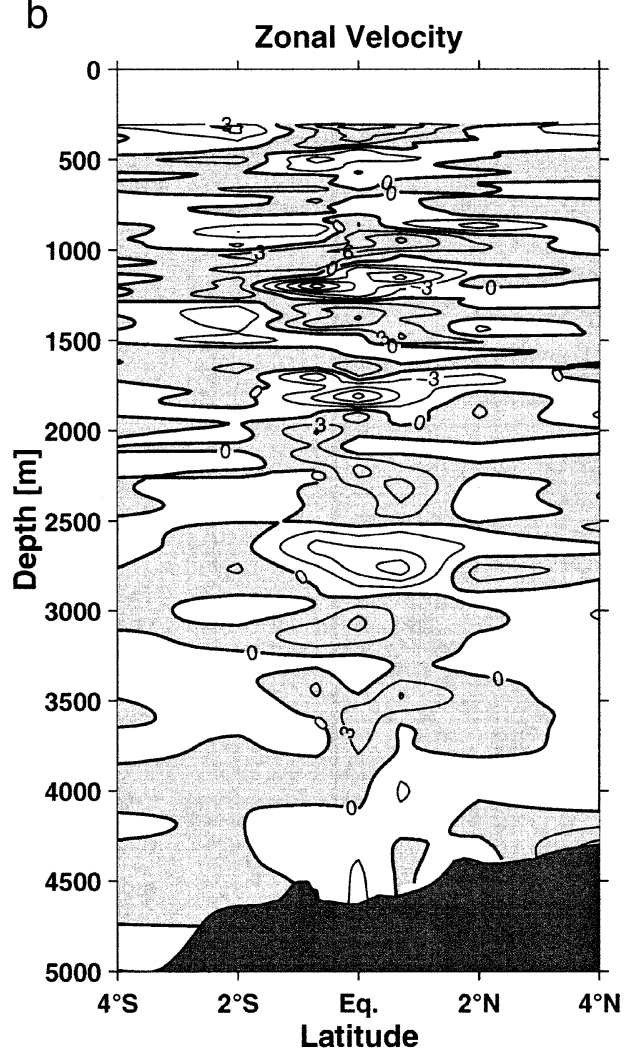

C

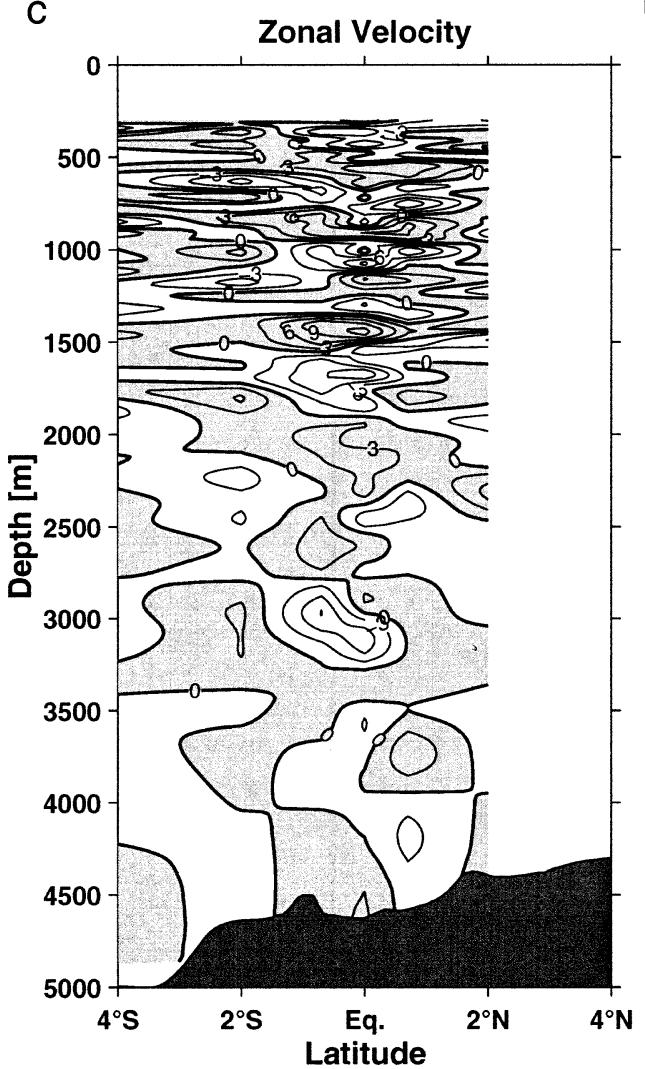

S088

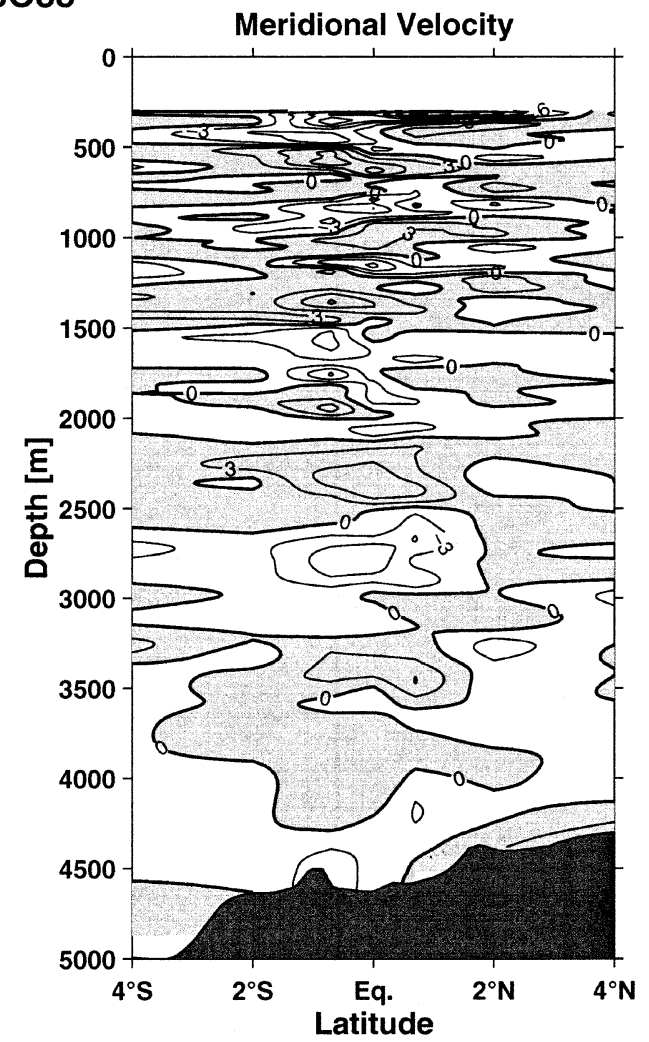

FR94

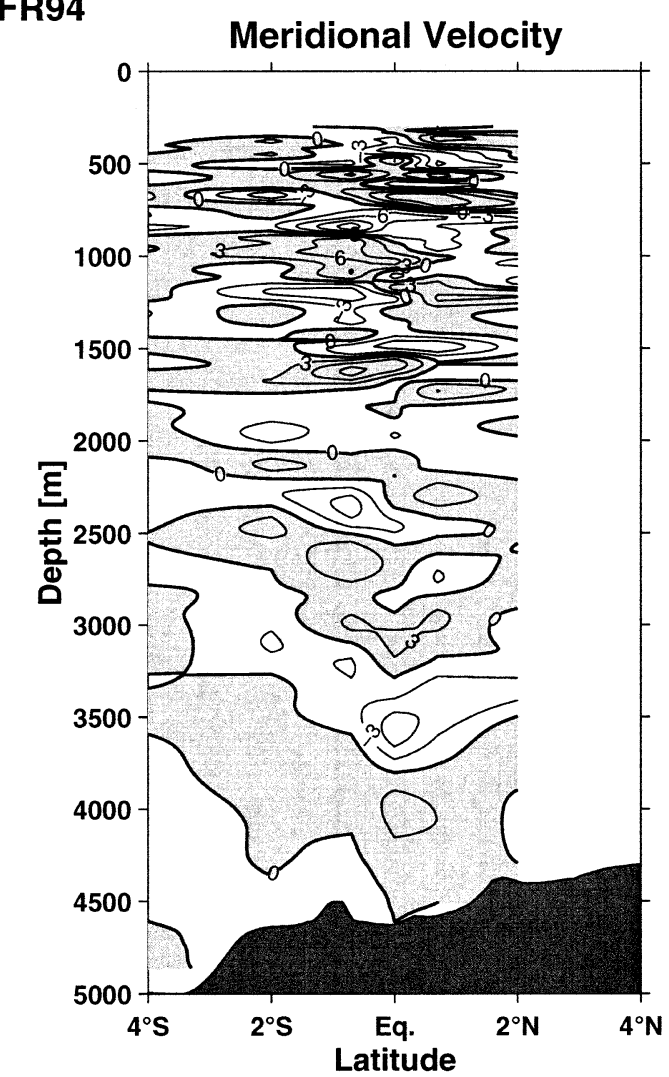

FIG. 3. (Continued) 
nantly eastward flow in January 1991 and July 1993 with zonal velocities exceeding $20 \mathrm{~cm} \mathrm{~s}^{-1}$. Eastward flow was also found between 400 and $700 \mathrm{~m}$ in September 1994. In the Pacific and Atlantic Oceans, this depth range is occupied by the westward-flowing Equatorial Intermediate Current (e.g., Gouriou and Toole 1993; Schott et al. 1995). Mooring data indicate the presence of westward flow here only during the monsoon transition periods (Reppin et al. 1999). A pronounced southward current of more than $20 \mathrm{~cm} \mathrm{~s}^{-1}$ also existed in July 1993 between 200 and $600 \mathrm{~m}$.

Below $1000 \mathrm{~m}$, the short vertical-scale features are superimposed on a low vertical mode current structure. There is a hint of low mode current reversal between January 1991 and July 1993. Zonal velocity was westward between 1000 and $3000 \mathrm{~m}$ in the SO88 and FR94 data collected during the SW monsoon season, whereas the flow was eastward during SO73. The meridional currents below $2000 \mathrm{~m}$ were predominantly northward during SO88 and FR94. Again, currents of opposite direction prevailed in the data collected during the NE monsoon. Of course, the temporal resolution of the dataset is not adequate to allow determination of the lowmode periodicity.

To emphasize the short vertical scales of the equatorial currents, the long vertical scale flows were removed from each velocity component of each profile by modal decomposition below 300-m depth. The calculation procedure is explained in chapter 4 . Mode 10 was chosen to separate low mode and high mode fluctuations to facilitate a comparison with other observations. The high-mode flow field of each cruise is contoured in Fig. 3. Most obvious in all three plots is the dominance of a few vertical modes in the zonal velocity component, leading to a distinct current structure that alternates sign with depth. Within $1^{\circ}$ of the equator, these zonal currents are also pronounced at lower depths. Meridional velocity also exhibits short vertical-scale variability, but amplitudes are larger in zonal velocity. At $2^{\circ}$ off the equator a reversal in zonal jet flow direction is frequently evident.

During SO73 in January 1991, the zonal velocity shows four deep jets at the equator below the thermocline to about $1800 \mathrm{~m}$ (Fig. 3a). The shallowest deep current flows westward between 850 and $1100 \mathrm{~m}$ and is followed by three alternating currents of similar vertical extent. Zonal velocities larger than $12 \mathrm{~cm} \mathrm{~s}^{-1}$ are associated with the cores of the jets. Meridional velocity between 900 and $1100 \mathrm{~m}$ is northward and changes direction at similar depths as the zonal velocity. Unlike the zonal current cores, meridional velocity maxima are not always positioned directly at the equator. Below $1800 \mathrm{~m}$ four more alternating zonal jets with wavelengths from 500 to $750 \mathrm{~m}$ extend to the ocean floor. The strongest of these centered at $3400 \mathrm{~m}$, flowed westward with velocity again exceeding $12 \mathrm{~cm} \mathrm{~s}^{-1}$. The increase of the jets' vertical scale is in accordance with buoyancy scaling.
A similar vertical distribution was found in the data collected during SO88 in July 1993 (Fig. 3b). An uppermost zonal jet was again located between 850 and $1100 \mathrm{~m}$, but directed eastward, opposite to the SO73 data. This flow reversal relative to the SO73 data continued in the jets located deeper in the water column. The core velocities of the zonal jets are less than observed during SO73. Note that at nearly all depths of deep jet cores, reversed velocities are found at $2^{\circ} \mathrm{S}$ and at $2^{\circ} \mathrm{N}$.

The vertical structure of the alternating currents collected during FR94 in September 1994 differed from the earlier observations (Fig. 3c). Generally, the vertical structure in both components is less organized as compared to the observations in January 1991 and in July 1993. Compared to the other two cruises, the amplitudes of the jets are smaller, most noticeably in the water column above $2000 \mathrm{~m}$. However, eastward flow is again found at $900 \mathrm{~m}$, followed by westward flow at about the same depth as during SO88.

\section{b. Low stratification layers}

The CTD data collected at $80.5^{\circ} \mathrm{E}$ show thick vertical layers of nearly uniform temperature and salinity. The difficulty of an objective separation of a profile into high versus low gradient sections has been addressed by Desaubies and Gregg (1981). In this study, we use data on a 1-db depth grid since the drop rate for the CTD was too high to obtain accurate data with a higher vertical resolution. For the following analysis, a low stratification layer is defined as having a depth interval larger than $15 \mathrm{~m}$ in which the mean vertical gradient of potential density vanishes within the measurement accuracies for salinity and temperature. This criteria can only hold for the upper $2000 \mathrm{~m}$ of the water column, since in deeper waters the mean vertical change of the potential density over $15-\mathrm{m}$ depth is smaller than the accuracy for potential density.

Figure 4 shows a density profile at the equator with two such low-stratification layers. Within the layer, both temperature and salinity are uniform within measurement accuracies. Using the CTD data collected during SO73, more than 100 such layers were identified. As an example, their distribution with depth and latitude is shown in Fig. 5. A similar distribution of the layers was obtained from the CTD data collected during SO88 and FR94. The layers concentrate within $1^{\circ}$ of the equator, but also exist close to Sri Lanka. At the equator, almost $10 \%$ of the analyzed water column has low stratification. This equatorial density fine structure during SO73 is located principally at depths around 750, 900, and 1300 $\mathrm{m}$ and it appears that most of the layers are located above or below the cores of the equatorial deep flow. Above $250 \mathrm{~m}$ in the thermocline, no low-stratification layers were observed. 


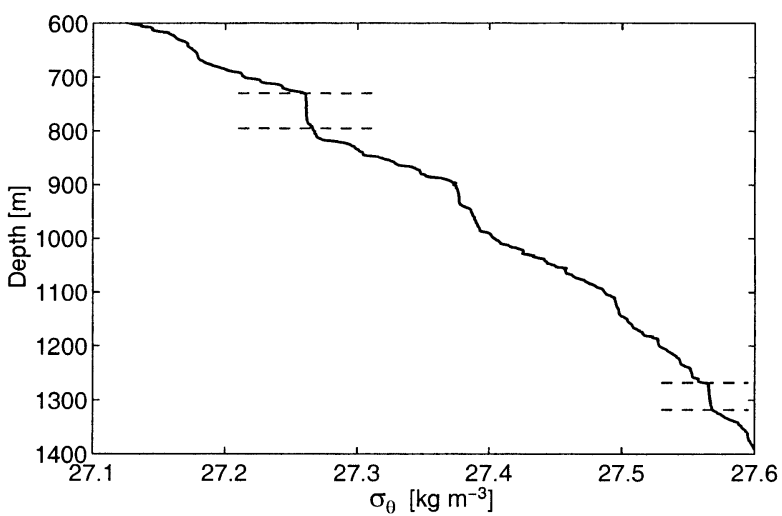

FIG. 4. Profile of potential density collected at the equator $\left(0^{\circ} 01.82^{\prime} \mathrm{N}\right)$ at $80.5^{\circ} \mathrm{E}$. Dashed lines serve as a setting for particularly large step structures.

\section{Scales of equatorial deep currents}

Two methods are commonly used to transform profiles of horizontal currents into vertical wavenumberamplitude space. In the first method, the horizontal velocities and the depth coordinate of the profiles are scaled with the local buoyancy frequency (WKBJ stretching). A Fourier decomposition is then used to transform the stretched profiles (Leaman and Sanford 1975). In the second method, the separated vertical differential equation

$$
F_{n}^{\prime \prime}(z)+\frac{N^{2}(z)}{c_{n}^{2}} F_{n}(z)=0
$$

is solved numerically. Here $F_{n}$ are the vertical velocity eigenfunctions for the $n$th mode, $c_{n}$ the eigenvalues, and $N$ the buoyancy frequency. The solutions define an orthonormal system. Mode amplitudes are then calculated by projecting the velocity profiles onto the modes. A comparison of both methods is given by Firing (1988). The limit of WKBJ stretching is its invalidity in the thermocline (e.g. O'Neill 1984). Thus, the top $300 \mathrm{~m}$ of the water column are usually excluded from Fourier decompositions.

The focus of our investigation is the deep equatorial currents and we thus exclude the top $300 \mathrm{~m}$ from our analysis. We choose modal decomposition as a tool to determine the vertical wavenumbers associated with the deep jets. For coherence and phase estimates the profiles were WKBJ-stretched and windowed in depth, so that Fourier decomposition resolves the vertical scales of the jets.

\section{a. Vertical scales}

Dynamical modes were calculated using a rigid-lid boundary condition and the mean buoyancy frequency profile obtained from all CTD profiles collected within $4^{\circ}$ of the equator. Calculation of the buoyancy frequency was done using the Fofonoff adiabatic leveling method

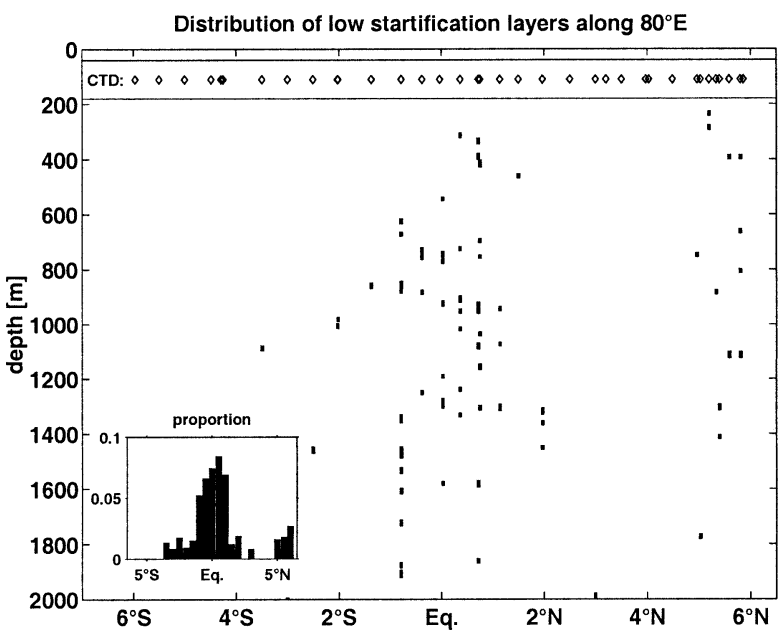

FIG. 5. Meridional distribution of low-stratification layers along $80^{\circ} \mathrm{E}$. Lines indicate the location and thickness of the layers. CTD stations are indicated by the diamonds at the top. The subplot shows the ratio of accumulated intervals with low stratification to the total depth of the sampled water column.

(Fofonoff 1985) with least squares fits over $20 \mathrm{~m}$. The eigenfunctions of Eq. (1) were differentiated to obtain the eigenmodes of the horizontal velocity components and then normalized to unit variance. The mode amplitudes were then determined by calculating the covariances between modes and velocity profiles.

When the top $300 \mathrm{~m}$ are omitted, the orthogonality of the vertical modes is lost. This is most severe for the first few modes, because most of their variance is contained in the upper water column. For modes larger than 10 , the departure in orthogonality is less than $20 \%$ for adjacent pairs and less than 5\% for all other pairs. In Fig. 3, the mode 10 and higher amplitudes obtained from the decomposition of the Pegasus profiles were mapped back onto the current structure.

Modal energy, defined here as the square of mode amplitude, for modes 8-25 are displayed in the upper panel of Fig. 6. In this presentation the amplitudes obtained from the profiles were squared and then averaged. Amplitudes of the first seven modes are not shown because they are not well represented due to the larger linear dependence of the calculation.

A peak in zonal velocity at mode 16 is very pronounced in the data from the equator. Together with modes 15 and 17 , this signal contains more than $12 \%$ of the total variance in the zonal component and more than $33 \%$ of the variance in modes above 10 . Due to its high energy level, we identify this mode as characterizing the vertical wavelength of the equatorial deep jets. The equivalent stretched-meter wavelength is 660 sm when a reference buoyancy frequency of $N_{0}=1$ cph is used. Summing the amplitudes of modes 15-17 yields a current structure with velocities exceeding 8 $\mathrm{cm} \mathrm{s}^{-1}$ in the cores of the jets. The meridional velocity also shows increased energy at mode 16 , which amounts 

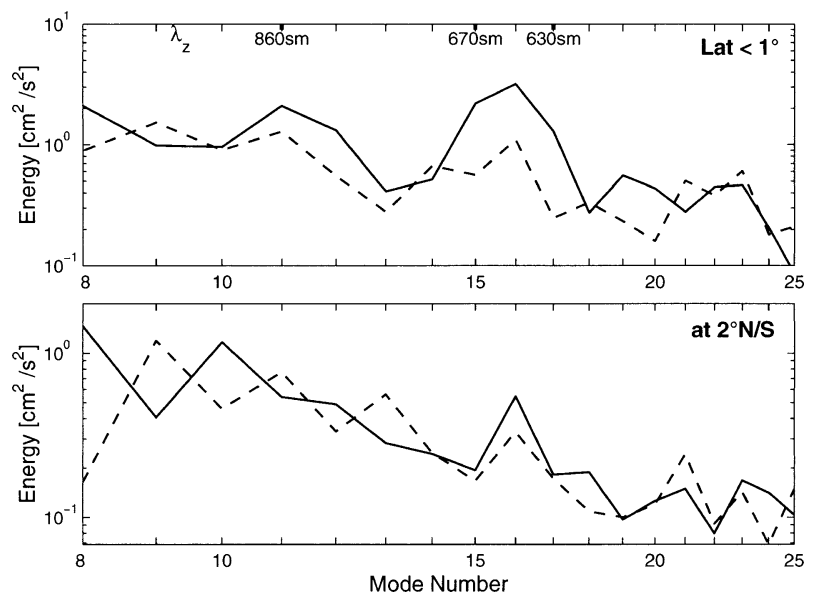

FIG. 6. Square of amplitudes obtained from modal decomposition of the Pegasus profiles (mode number 8-25) of SO73 and SO88 collected within (top) $1^{\circ}$ of the equator and (bottom) at $2^{\circ}$ off the equator. The solid line is the zonal and the dashed line the meridional velocity.

to about $25 \%$ of the zonal value. Here, the signal contains $6 \%$ of the total meridional variance and $19 \%$ of the variance when looking at modes 10 and higher. Both velocity components show a secondary maximum centered at mode 11 . The equivalent stretched-meter wavelength is $860 \mathrm{sm}$ and the sum of mode 11 and 12 has maximum velocities of $3 \mathrm{~cm} \mathrm{~s}^{-1}$ at the equator. Energies at $2^{\circ}$ off the equator are generally lower, but there also exists a small peak at mode 16 .

Figure 7 shows the energy distribution for modes 2575. Enhanced energy is found at modes 32 and 33, twice the modenumber associated with the deep jets, and at mode 27. Meridional energies at these bands are also slightly enhanced, but again less than the zonal counterpart. The mode 32 maximum at $330 \mathrm{sm}$ may account for small deviations of the dominant modal structure. However, the influence on the deep jet structure and its variance contribution is small (2.9\% of total variance). At very short vertical scales, an excess of meridional mode energy is seen in the modal range from 60 to 66, corresponding to a band in vertical wavelength from 152 to $171 \mathrm{sm}$. This signal contributes $2.4 \%$ to the total variance of the vertical meridional structure.

The eastward jet scales reported by O'Neill and Luyten (1984) compare well with the results from our modal decomposition for $80.5^{\circ} \mathrm{E}$. Their most energetic peak centered at $720 \mathrm{sm}$ lies between our maxima, modes 11 and 16 . Our two secondary maxima at modes 27 and 32 correspond to their 360 -sm wavelength maximum. However, zonal energy at mode 16 was high in the SO73 and SO88, but only slightly enhanced in September 1994, suggesting that energies at these scales are subject to temporal variability. This may explain the absence of energetic maxima in the velocity autospectra reported by Ponte and Luyten (1990).

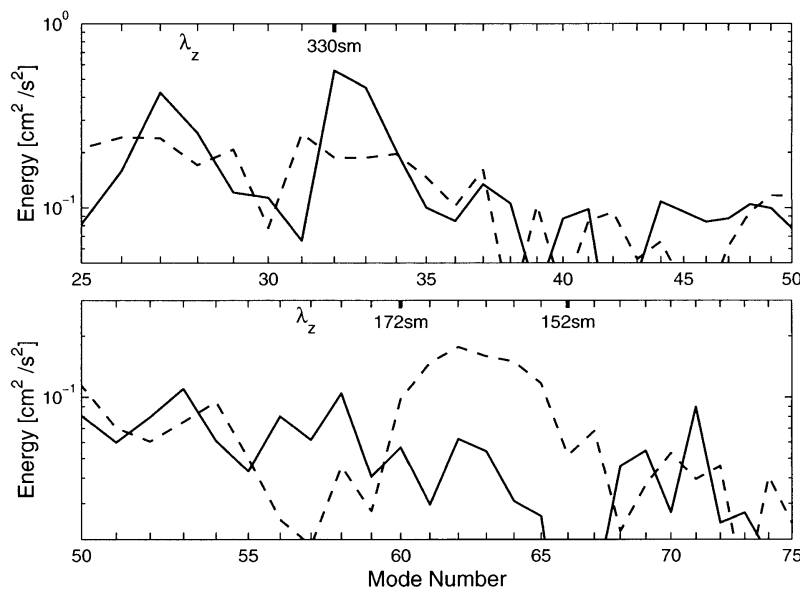

FIG. 7. Square of amplitudes obtained from modal decomposition of the Pegasus profiles collected within $1^{\circ}$ of the equator for mode numbers (top) $25-50$ and (bottom) $50-75$. The solid line is the zonal and the dashed line the meridional velocity.

\section{b. Coherence and phase estimates}

Having established the dominant vertical wavelength of the deep jets (mode 16, $660 \mathrm{sm}$ ), we derived coherence $(\gamma)$ and phase estimates, in particular, correlation between vertical displacement and the individual velocity components to explore the dynamical nature of the jets.

The deviation of potential density at a particular depth from the time- and horizontally averaged potential density, divided by the 400-m lowpass-filtered vertical gradient of the averaged potential density, was used to estimate vertical displacement. The meridional averaging interval was $\pm 0.5^{\circ}$ about the transponder positions. The calculation had to be restricted to the depth range between 300 and $2000 \mathrm{~m}$, because only few CTD casts reached below 2000-m depth. Averages were calculated using the CTD data from the four sections. Each ensemble included 10-13 potential density profiles. WKBJ-scaling of the velocity components and vertical displacement was performed using the mean buoyancy profile from each cruise mentioned above. The methodology applied is identical to that described by Eriksen (1981).

The stretched profiles were spline interpolated onto a regular vertical grid of $6.2 \mathrm{sm}$ to resolve the $660-\mathrm{sm}$ band. Coherence estimates were calculated between velocity and vertical displacement as well as between the horizontal velocity components. Prior to Fourier decomposition, up and down velocity profiles were averaged and a 1/2-cosine taper was applied to the first and the last $10 \%$ of each profile.

As will be discussed in the next section, the phase relation between horizontal velocity and vertical displacement may be used to distinguish between Rossby and Kelvin waves. Within $1^{\circ}$ of the equator, coherence estimates between vertical displacement and horizontal velocity at 660 -sm band are significantly different from 
TABLE 1. Coherence $(\gamma)$ and phase of vertical displacement $(\zeta)$ and horizontal velocity of the 660 -sm band. The subscript $<1^{\circ}$ refers to all profiles within $1^{\circ}$ of the equator, $0.7^{\circ}$ refers only to the profiles collected at $0.7^{\circ}$ off the equator, $2^{\circ}$ only to those from $2^{\circ}$ off the equator. Values in parentheses represent the critical value of zero significance at $95 \%$ confidence. The $95 \%$ confidence interval for phase is indicated by \pm .

\begin{tabular}{lcc}
\hline \hline & Coherence & Phase $\left(^{\circ}\right)$ \\
\hline$U_{<1^{\circ}}-\zeta_{<1^{\circ}}$ & $0.60(0.56)$ & $51 \pm 45$ \\
$U_{2^{\circ}} \zeta_{2^{\circ}}$ & $0.71(0.72)$ & $-99 \pm 53$ \\
$V_{<1^{\circ}}-\zeta_{<1^{\circ}}$ & $0.29(0.56)$ & - \\
$U_{0.7^{\circ}}-U_{2^{\circ}}$ & $0.77(0.67)$ & $-138 \pm 36$ \\
\hline
\end{tabular}

zero for the zonal component (Table 1). Zonal velocity leads vertical displacement by up to $\pi / 2$. Coherence of the $U$ and $\zeta$ pairings from $2^{\circ}$ off the equator is just below the $95 \%$ confidence level for the wavelength of $660 \mathrm{sm}$. Here, zonal velocity lags vertical displacement by about $\pi / 2$.

To investigate the geometry of the jets, cross-spectral calculations between horizontal velocity components were carried out using full depth buoyancy scaled profiles. Within $1^{\circ}$ of the equator, significant coherence at zero phase was found between all zonal velocity profiles from each cruise for all wavelengths larger than 540 sm. Velocity comparisons between $0.7^{\circ}$ and $2^{\circ}$ off the equator for the 660-sm band were again only significant for zonal velocity. Here, a phase difference of $-138^{\circ}$, close to $-\pi$, was obtained, indicating a flow reversal of the deep jets at $2^{\circ}$ off the equator. This can also be seen in the data displayed in Fig. 3.

A feature of the Hua et al. (1997) theory, which explains the jets as a by-product of angular momentum mixing, is the high correlation of the velocity components at the vertical scales of the deep jets. In their model, the vertical position of an eastward jet coincides with meridional flow toward the equator and vice versa. To explore this hypothesis, coherence was calculated for various pairings of zonal and meridional velocity at different latitudes and cruises. However, no significant coherence was obtained between the velocity components to support the theory. Though not statistically significant, elevated $U-V$ coherence was found within $1^{\circ}$ of the equator at the 660 -sm band, when data from each cruise were compared separately. Here, the phase differences between the velocity components varied for each cruise.

Coherences were also estimated for the zonal velocity between the three different cruises: The methodology is known as the drop-lag coherence (DLC) estimate (Hayes 1975). Here it is assumed that the processes are stationary; so the cross spectra at a given vertical wavenumber depend only on the time difference between the profiles. Figure 8 (bottom) shows the drop-lag coherence phase between SO73 and SO88 for the three transponder locations within $1^{\circ}$ of the equator. The pairings for all three cruises are summarized in Table 2. Zonal velocities are coherent at the 660-sm wavelength despite
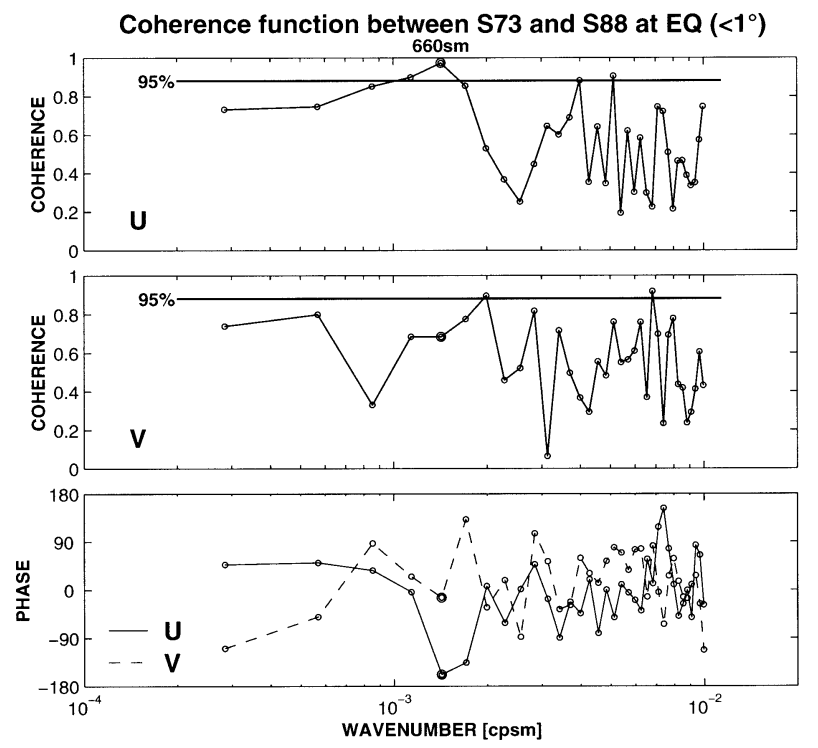

FIG. 8. Coherence of horizontal velocities collected within $1^{\circ}$ of the equator between SO73 and SO88; (top) zonal and (center) meridional components (bottom) phase of the zonal and meridional components. A straight line is drawn at the $95 \%$ confidence zero significance level.

the large differences in sample time. Phase lags $\left(-149^{\circ}\right.$ and $175^{\circ}$ ) indicate that the vertical positions of the jets differ among the cruises, but are consistent with a phase reversal from January 1991 to July 1993 and to September 1994. This temporal phase reversal of the deep jets has also been suggested in section 3 .

The poor temporal resolution of the dataset does not permit a quantification of the deep jets' period. O'Neill and Luyten (1984) found a timescale much longer than 1 month for the deep jets in the western Indian Ocean. The DLC phase reversal $\left(-149^{\circ}\right)$ from January 1991 to July 1993 is inconsistent with the semiannual period that one might expect, given the dominant semiannual windforcing.

\section{Equatorial waves}

Our basic findings of the equatorial deep jets are interpreted in terms of free linear equatorial waves. For a detailed model description, the reader is referred to Moore and Philander (1977) and Eriksen (1980). There are two classes of low-frequency wave solutions on the

TABLE 2. Drop-lag coherence and phase for the 660-sm band. Critical value of zero significance at $95 \%$ confidence based on three degrees of freedom for coherence is 0.881 .

\begin{tabular}{lccc}
\hline \hline $\begin{array}{l}\text { Time interval } \\
\text { cruises }\end{array}$ & $\begin{array}{c}\text { Jan 91-Jul 93 } \\
\text { SO78-SO88 }\end{array}$ & $\begin{array}{c}\text { Jul 93-Sep 94 } \\
\text { SO88-FR94 }\end{array}$ & $\begin{array}{c}\text { Jan 91-Sep 94 } \\
\text { SO73-FR94 }\end{array}$ \\
\hline Zonal coherence & 0.975 & 0.904 & 0.893 \\
Zonal phase $\left(^{\circ}\right)$ & $-149 \pm 19$ & $-42 \pm 41$ & $175 \pm 45$ \\
Time lag (days) & 930 & 412 & 1342 \\
\hline
\end{tabular}



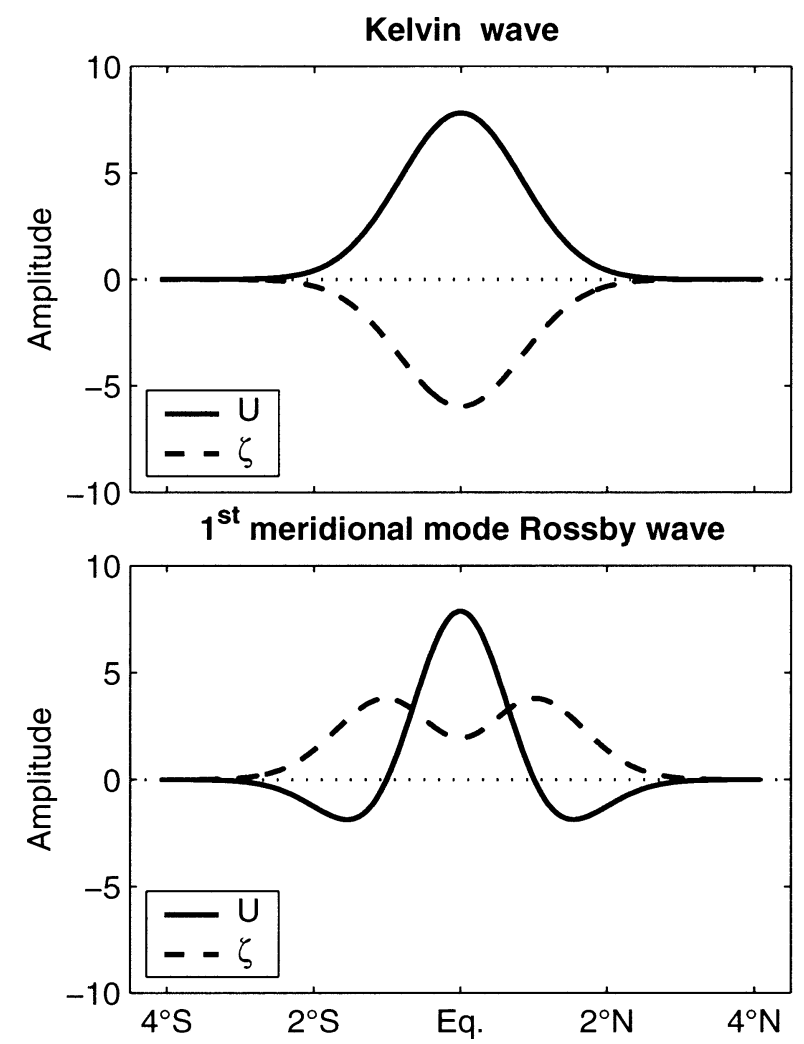

FIG. 9. Meridional distribution of zonal velocity and vertical displacement of a (top) Kelvin wave and (bottom) a first meridional mode Rossby wave. The amplitude distribution of vertical displacement is shown as it would be found $1 / 4$ of a wavelength below the maximum in zonal velocity.

equatorial $\beta$ plane: equatorial Rossby and Kelvin waves. We relate both model solutions to the observations.

Kelvin waves have a unidirectional meridional distribution of zonal velocity for a given vertical mode (Fig. 9), which conflicts with the observed reversal of zonal flow at $2^{\circ}$ off the equator. Furthermore, vertical displacement of these waves has a maximum $1 / 4$ of a wavelength above the cores of zonal velocity as indicated in Fig. 9. At two degrees off the equator, a comparable phase relationship between $U$ and $\zeta$ was found. However, due to the smaller meridional trapping scale of Kelvin waves compared to that of Rossby waves, a coherence at this latitude cannot be expected. Within $1^{\circ}$ of the equator, cross-spectral analysis showed that zonal velocity leads vertical displacement, which is again not consistent with a Kelvin wave description.

Rossby waves have a complicated meridional structure with reversals in phase of zonal velocity at various latitudes depending on the meridional mode number. Due to the observed symmetry of zonal velocity about the equator, only odd meridional mode Rossby waves are consistent with the observations. For each meridional mode, there are two solutions for a specified frequency: dispersive (short) and nondispersive (long) Rossby waves. Dispersive Rossby waves are dominated
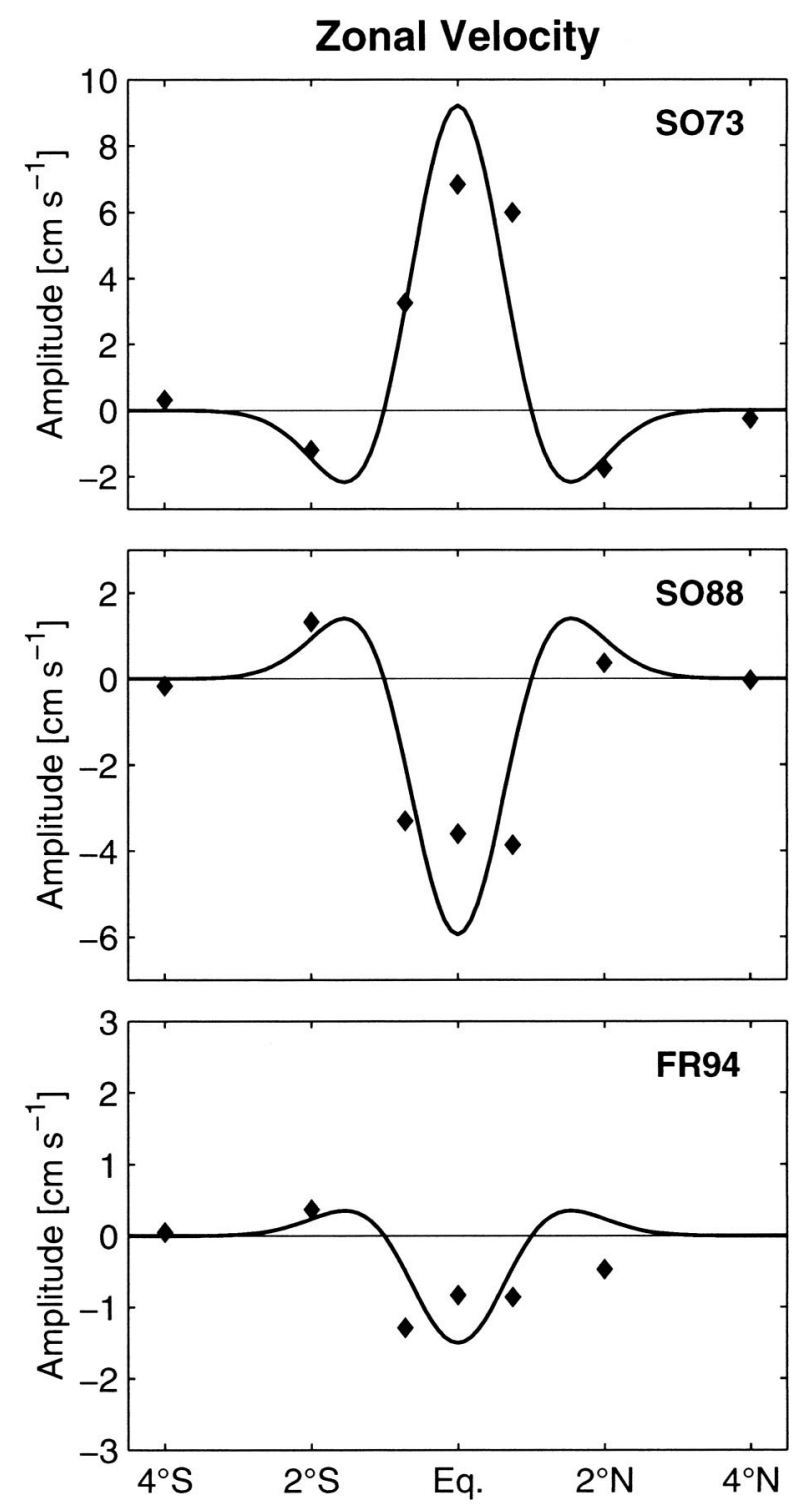

FIG. 10. Least squares fit of the meridional structure function of zonal velocity for a 1st meridional mode, 16 th vertical mode Rossby wave to the amplitude distribution obtained from the modal decomposition (diamonds) for the same vertical mode for each cruise; (top) SO73, (middle) SO88, and (bottom) FR94.

by meridional flow. In contrast, zonal current amplitudes are much larger than meridional current amplitudes for nondispersive Rossby waves, in agreement with the observations.

The meridional distribution of zonal velocity for the first meridional mode nondispersive Rossby wave predicted by linear theory agrees with the observations (Fig. 10). For the 16th vertical mode, zonal velocity reverses at about $1^{\circ}$ off the equator, which can account for the observed phase reversal of the zonal flow. In the model, this zonal flow reversal also causes a change of sign in the phase relation between zonal velocity and vertical displacement (Fig. 9). Within $1^{\circ}$ of the equator, 
zonal velocity leads vertical displacement by $\pi / 2$ and poleward of $1^{\circ}$ vertical displacement leads zonal velocity. The results from cross-spectral analysis between $U$ and $\zeta$ are consistent with the model predictions: within $1^{\circ}, U$ leads $\zeta$ in phase and at $2^{\circ}$, the phase had reversed. The first meridional mode nondispersive Rossby wave is thus consistent with all significant results from crossspectral analysis.

To test the hypothesized relationship between the lowstratification layers and a first meridional mode Rossby wave, we calculated the meridional and vertical distribution of strain for a nondispersive Rossby wave of 16th vertical mode and compared the distribution to the position and to the vertical extent of the low-stratification layers. Strain is the vertical derivative of vertical displacement; positive strain can be responsible for layers whereas negative values cause sheets in the density structure.

About $60 \%$ of the low stratification was found in positive strain regions compared to $40 \%$ found in negative strain regions. Most of the layers with a vertical extent larger than $30 \mathrm{~m}$ (9 out of 11 layers in the SO73 data) were correlated with elevated positive strain regions. However, no obvious correlation was found for the smaller low-stratification layers. This indicates that other processes may also contribute to their generation.

\section{Shear and strain spectra}

Spectral estimates of shear and strain were calculated for the depth range between 800 and $1800 \mathrm{~m}$ where the deep jets are most pronounced. The variation in mean buoyancy frequency is small below $700 \mathrm{~m}$, favoring the validity of the WKBJ approximation. To increase statistical reliability, the CTD and Pegasus datasets were grouped into three bins. All profiles collected within $1^{\circ}$ of the equator, between $1.5^{\circ}$ and $2.5^{\circ}$ and between $3.5^{\circ}$ and $4.5^{\circ}$ off the equator form separate ensembles. Within $1^{\circ}$ of the equator, 24 CTD profiles and 13 Pegasus profiles are available. The ensemble at $2^{\circ}$ consists of 15 CTD casts and 14 Pegasus profiles and at $4^{\circ}$ off the equator, $12 \mathrm{CTD}$ and 10 velocity measurements were used. The results from the analysis reported below do not change when both hemispheres are treated separately.

Profiles of velocity components were WKBJ scaled with the mean buoyancy frequency of $1.29 \mathrm{cph}$ and then spline interpolated onto a 15 -sm vertical grid. Due to the stretching of the depth coordinate and the filtering of velocity, wavenumber resolution is limited to $k_{z}<$ $0.013 \mathrm{cpsm}$. Velocity component spectra were computed using prewhitened (by first differencing) and detrended profiles. A 1/2-cosine taper was also applied to the first and the last $10 \%$ of each profile prior to Fourier decomposition. The resulting coefficients were then recoloured, normalized, and squared. Shear spectra were calculated from velocity spectra by adding both velocity component spectra and multiplying with $\left(2 \pi k_{z}\right)^{2}$.

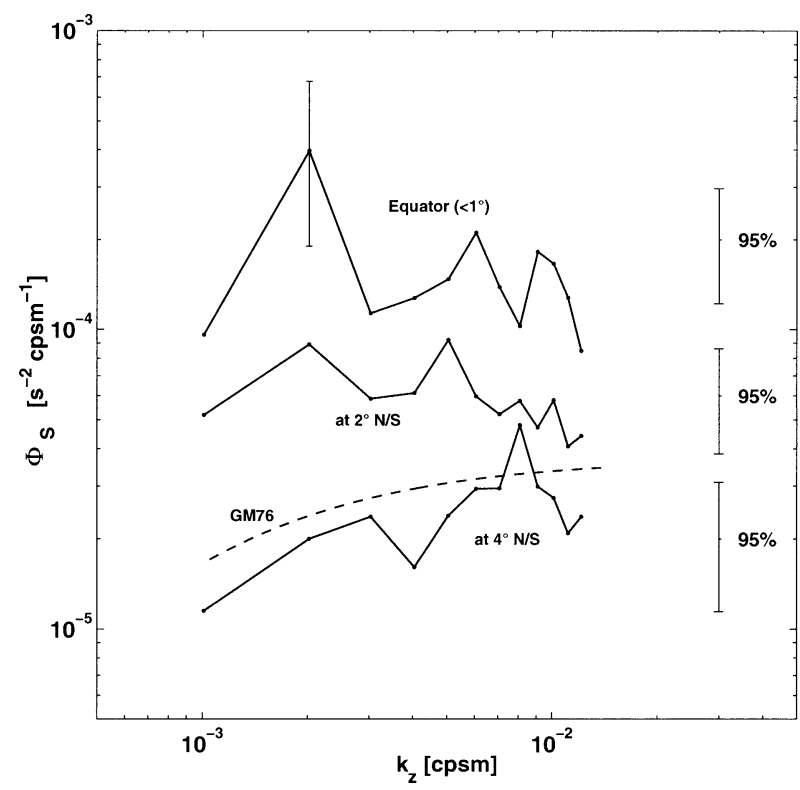

FIG. 11. Shear spectra of WKB-scaled Pegasus profiles for the depth range from 800 to $1800 \mathrm{~m}$ and for different ensemble latitudes off the equator. The GM76 shear spectra is indicated by the dashed line. For the deep jets shear contribution, up- and downcasts are not independent realizations and the $95 \%$ confidence intervals are displayed separately.

Strain spectra were estimated from temperature spectra for each latitude band (e.g., Eriksen 1981). The CTD profiles collected south of $2^{\circ} \mathrm{S}$ show intrusions of cold low saline water from the Indonesian Throughflow in the upper $1000 \mathrm{~m}$. Therefore we restricted the analysis to below $1100-\mathrm{m}$ depth. A linear average temperature gradient was found for all ensemble latitudes between 1100 and $1600 \mathrm{~m}$. Temperature spectra were WKBJ scaled before the mean temperature profile was subtracted. Mean buoyancy frequency in the selected depth range was $N_{0}=1.24 \mathrm{cph}$. Here, stretching of the depth coordinate creates uncertainties at wavenumbers larger than $k_{z}>0.25 \mathrm{cpsm}$. Spectral manipulations are identical to the ones used in the calculation of shear spectra. To obtain statistical confidence, segments of 256 values were used for the Fourier transformation, resulting in two independent spectral estimates for each profile. No overlapping segments were used. Strain spectra were then obtained from temperature displacement spectra by multiplying with $\left(2 \pi k_{z}\right)^{2}$ and normalizing with the square of the average temperature gradient.

\section{a. Shear}

Shear spectra from the different ensemble latitudes are displayed in Fig. 11. Spectral levels at the equator are more than five times higher than those at $4^{\circ}$ and about twice as high as at $2^{\circ}$ off the equator (Table 3 ). Maximum spectral energy at the equator is found at $k_{z}$ $=0.002 \mathrm{cpsm}$ indicating the shear contribution from the deep jets. The shear spectral levels are flat over the 
TABLE 3. Average shear spectral density and cutoff wavenumber calculated from (Eq. 2) using the mean buoyancy frequency over the analyzed column of $N_{0}=1.29 \mathrm{cph}$.

\begin{tabular}{ccc}
\hline \hline $\begin{array}{c}\text { Latitude } \\
(\mathrm{N} / \mathrm{S})\end{array}$ & $\begin{array}{c}\text { Average shear spectral } \\
\text { density } \Phi_{s}\left(\mathrm{~s}^{-2} \mathrm{cpsm}^{-1}\right)\end{array}$ & $\begin{array}{c}\text { Cutoff wavenumber } \\
k_{c}(\mathrm{cpsm})\end{array}$ \\
\hline$<1^{\circ}$ & $1.45 \times 10^{-4}$ & 0.024 \\
At $2^{\circ}$ & $6.32 \times 10^{-5}$ & 0.055 \\
At $4^{\circ}$ & $2.70 \times 10^{-5}$ & 0.13 \\
GM76 & $3.06 \times 10^{-5}$ & 0.10 \\
\hline
\end{tabular}

whole resolved wavenumber range within the $95 \%$ confidence limits. The shear spectrum of the internal wave model by Garrett and Munk (1975), as modified by Cairns and Williams (1976), hereafter GM76, was computed following Gregg and Kunze (1991). The spectrum was adjusted to the mean buoyancy frequency over the depth interval used here. Spectral energy at $4^{\circ}$ compares well in wavenumber distribution to the GM76 model, but has a slightly lower spectral level.

The canonical shear spectrum (Gargett et al. 1981) is flat for low wavenumbers with a change in slope to $k_{z}^{-1}$ observed at larger wavenumbers. The wavenumber $k_{c}$ marks the transition scale of the internal wave field to the roll-off range, where in the saturation hypothesis the internal waves become unstable (Dewan and Good 1986) or wave energy is shifted to higher wavenumbers by strong Doppler shifting (Hines 1991). The transition wavenumber was defined by Polzin et al. (1995) as

$$
\int_{0}^{k_{c}} \Phi_{s} d k_{z}=0.7 N^{2}
$$

For comparison with other measurements, $k_{c}$ was calculated by evaluating (2) using the averaged spectral density over the resolved wavenumber range $(0.001-$ $0.013 \mathrm{cpsm}$ ). The shear contribution of the deep jets was excluded from the calculation. Instead, the mean value over the resolved wavenumber interval was used. The results are listed in Table 3. Although the velocity measurements do not resolve larger wavenumbers, the determined cutoff wavenumber agrees well with the transition wavenumber resolved by the strain estimates (Fig. 12).

Gregg et al. (1995) report cutoff wavenumbers of $k_{c}$ $\approx 0.03 \mathrm{cpm}$ (not stretched) at the equator near $140^{\circ} \mathrm{W}$ and $k_{c} \approx 0.05 \mathrm{cpm}$ at $156^{\circ} \mathrm{E}$ determined from multiscale profiler data between 640 and $990 \mathrm{~m}$ within the Pacific deep jets. In comparison, the spectral levels of the internal wave field in the Indian Ocean are twice as high as these in the Pacific. However, with the poor data coverage in either ocean in space and time, this result is not conclusive.

\section{b. Strain}

The underlying assumption in the calculation of vertical displacement, and subsequently strain, is that perturbations of the background stratification are due to

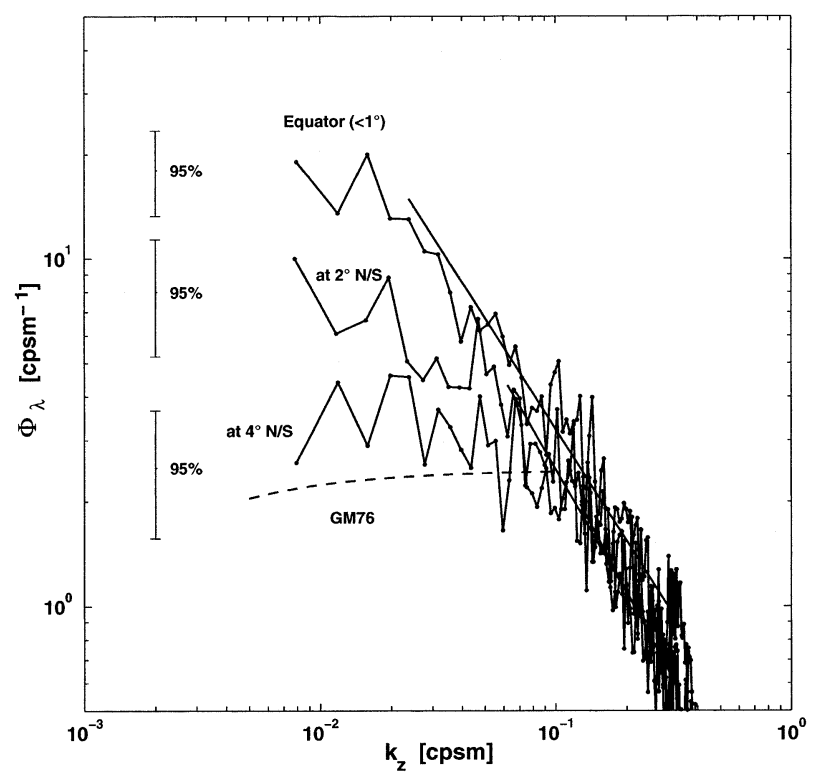

FIG. 12. Strain spectra of WKB-scaled CTD profiles for different ensemble latitudes off the equator in the depth range from 1100 to $1600 \mathrm{~m}$. Dashed line indicates GM76 model spectra. A least squares fit of the roll-off range is indicated by the straight lines.

internal waves only, not to vertical mixing or true fine structure. Although this assumption seems to hold for off-equatorial ensembles, our observations indicate that mixing is enhanced within $1^{\circ}$ of the equator (see next section). To find possible perturbation scales by mixing events, the resorting technique by Thorpe (1977) was applied to the potential density profiles to estimate overturning scales at the equator. Within the low-stratification layers, Thorpe displacements exceed $20 \mathrm{~m}$. The scheme for identifying overturns from Galbraith and Kelly (1996) was applied to all profiles of the equatorial ensemble. The probability density functions of the Thorpe displacements in the CTD casts were compared to random noise to yield a threshold for the CTD characteristics and local hydrographic properties. Although the accuracy of the CTD meets the World Ocean Circulation Experiment (WOCE) standard, overturning scales are not sufficiently large to be detected above CTD measurement noise, consistent with the results of Galbraith and Kelly (1996).

Figure 12 shows the spectra of internal wave strain versus vertical wavenumber in the depth range from 1100 to $1600 \mathrm{~m}$ for the different ensemble latitudes. The GM76 strain spectrum was computed as above. The general behavior of the spectra in Fig. 12 can be described as being white for small wavenumbers and then falling off as $k_{z}^{-1}$ toward higher wavenumbers. As for shear, the spectral level at the equator is about five times higher than at $4^{\circ}$ and about two to three times higher than at $2^{\circ}$ for $k_{z}>0.01 \mathrm{cpsm}$. The $4^{\circ}$ spectrum is close to GM76 levels only for $k_{z}<0.01 \mathrm{cpsm}$ and elevated by a factor of about 1.5 for larger wavenumbers. This implies that shear to strain ratios are smaller at the equa- 
tor than suggested by GM76. GM76 obtained a ratio of three, whereas a value of about two is obtained at all ensemble latitudes. The least squares fits in the roll-off range are $k_{z}^{-1.1}$ for the equatorial spectrum and $k_{z}^{-1.2}$ for the $2^{\circ}$ and $4^{\circ}$ spectra, the two latter fits match each other exactly (Fig. 12). In contrast, the equatorial roll-offrange is shifted to larger wavenumbers. Gregg et al. (1995) report a similar behavior of strain spectra calculated from equatorial CTD profiles from $156^{\circ} \mathrm{E}$, but the slope in the roll-off-range in their calculations is much larger than our data suggest. The lower wavenumber limit of the fit was chosen on the basis of the transition wavenumber calculated from shear variance.

\section{Dissipation estimates}

Kunze et al. (1990), hereafter KWB, provided a parameterization for dissipation rates based on Richardson number, valid only in unstable events

$$
\langle\epsilon\rangle=f r \Delta z^{2}\left\langle\left(\frac{S^{2}-4 N^{2}}{24}\right)\left(\frac{S-2 N^{2}}{4}\right)\right\rangle .
$$

Here $\Delta z$ is the vertical derivative scale and $f r$ the unstable fraction of the water column. The left term in the averaging bracket represents the available kinetic energy in a super-critical linearly stratified shear flow, which is dissipated as turbulent fluxes to decrease the shear below critical. The right term is the growth rate of Kelvin-Helmholtz billows (Hazel 1972). Polzin (1996) examined (3) over a broad range of internal wave environments at subtropical latitudes. He found that the KWB turbulence parameterization matched his dissipation data to within a factor of 2. Similar agreement is reported by Peters et al. (1995), who compared measured dissipation rates to shear and buoyancy data collected in the equatorial Pacific in the depth range from 100 to $300 \mathrm{~m}$.

The accuracy of the KWB parameterization critically depends on the resolved length scales. Polzin (1996) reports that the shear variance $\left(\left\langle S^{2}\right\rangle\right)$ of the observations must be larger than the square of mean buoyancy frequency $\left(\bar{N}^{2}\right)$ in order to achieve consistent estimates. The Pegasus data collected within $1^{\circ}$ of the equator from 500 to $2000 \mathrm{~m}$ have a vertical resolution of only $\left\langle S^{2}\right\rangle$ $=0.40 \bar{N}^{2}$ when calculating gradients on a basis of $\Delta z$ $=20 \mathrm{~m}$. For the four profiles collected at the equator shear resolution is enhanced, being $\left\langle S^{2}\right\rangle=0.67 \bar{N}^{2}$. However, this resolution still does not meet the above consistency requirement, although most of the internal wave variance is resolved by the measurements. This is indicated by the transition wavenumber determined from strain at the equator $\left(k_{c} \approx 0.0125 \mathrm{cpsm}\right)$. Away from the equator, shear variance is lower by at least a factor 2 . Therefore, we restrict the analysis to within $1^{\circ}$ of the equator.

Equation (3) was evaluated using least squares fits of the buoyancy frequency and shear calculated over suc- cessive depth intervals of $\Delta z=20 \mathrm{~m}$. The calculations were made for the interval between 500 and $2000 \mathrm{~m}$. Here, the mean buoyancy frequency is $\bar{N}=1.24 \mathrm{cph}$. Following Polzin (1996), we used a Richardson number lower than 0.33 as a criterion for instability. Unstable regions were found for $6.3 \%$ of the water column ( $\mathrm{fr}$ $=0.063)$. On the profiles collected directly at the equator, instability was found for $11 \%$. An estimate of the mean dissipation rate of $\bar{\epsilon}=7.5 \times 10^{-10} \mathrm{~W} \mathrm{~kg}^{-1}$ was obtained using the profiles collected during SO73 and SO88 from each transponder pair located at $0.7^{\circ} \mathrm{S}$, $0.7^{\circ} \mathrm{N}$, and at the equator. Mean inferred dissipation from only the equatorial transponder pair yields $\bar{\epsilon}=19$ $\times 10^{-10} \mathrm{~W} \mathrm{~kg}^{-1}$. Up- and downcasts agree within a factor two of each other, except for one equatorial cast, where the downcast has a dissipation rate enlarged by a factor 6. That particular profile shows very large inferred dissipation rates $\left(\epsilon>1000 \times 10^{-10} \mathrm{~W} \mathrm{~kg}^{-1}\right)$ over $20 \mathrm{~m}$ at about $1300-\mathrm{m}$ depths within a low stratification layer. Calculations with varying $\Delta z=10,15$, $25 \mathrm{~m}$ for buoyancy frequency estimates differed by less than $20 \%$ of the values above. Depth displacements between the buoyancy frequency and the shear profile of up to $20 \mathrm{~m}$ were also evaluated, but these did not affect the results.

Figure 13 shows a typical example of the depth distribution of Richardson numbers and inferred dissipation rates of one pair of equatorial CTD and Pegasus profiles. Indeed, we find most of the unstable depth intervals to be associated with the low stratification layers. The vertical resolution of the velocity profiles does not permit the determination of the small-scale shear contribution to dissipation and a possible adjustment of shear within the low-stratification layers cannot adequately be resolved. However, the large spectral level of shear at resolved scales and the cutoff wavenumber determination from strain spectra demonstrate that the contribution of larger wavenumbers to shear variance is of minor importance.

An error assessment for the inferred dissipation values given above is difficult and will not be attempted. Our study suffers, of course, from the fact that the velocity and density were not measured simultaneously at exactly the same position. The horizontal and temporal difference of the two instruments during data acquisition could be as large as $1.5 \mathrm{~km}$ and $30 \mathrm{~min}$. Midlatitude abyssal internal waves show horizontal spatial coherency scales of 15-20 km (Sanford 1991). Coherence of most of the equatorial internal wave field is known to be strongly reduced (Eriksen 1980, 1985). However, the good agreement between up- and downcast dissipation estimates (except for one profile) does put some confidence on our calculations. Measurement error of the Pegasus data may also contribute and lead to an overestimation of the dissipation rate. Attempts to quantify the error in velocity have been unsuccessful, because it could not be distinguished from variability caused by internal waves (Send 1994; Send et al. 1996). Another 

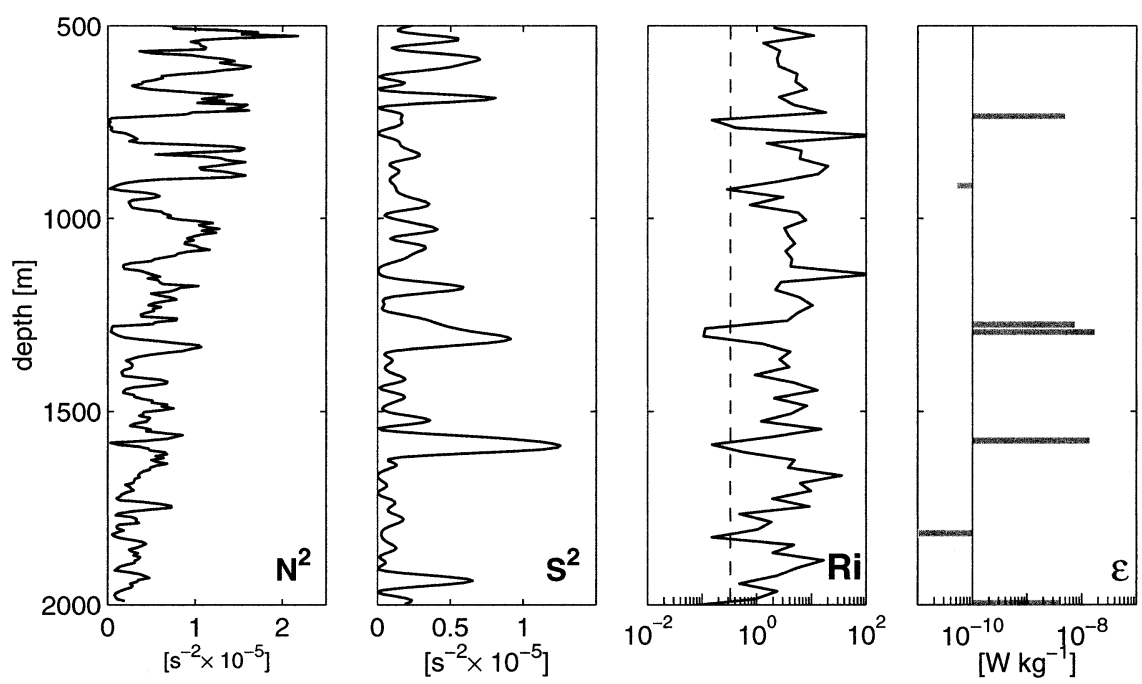

Fig. 13. Vertical profile of (left to right) buoyancy frequency $\left(N^{2}\right)$, vertical shear $\left(S^{2}\right)$, Richardson number (Ri), and dissipation $(\epsilon)$ estimates based on 20 -m vertical gradient.

source of uncertainty is inherent in the method itself. Both terms in the KWB parameterization have not been explored in the environment of deep equatorial jets and its validity there has not been proven.

In summary, the above results indicate that abyssal mixing at the equator is slightly elevated compared to mixing over abyssal plains at midlatitudes (e.g., Kunze and Sanford 1996). These results contrast with direct dissipation measurements within the deep jets of the Pacific and Atlantic Ocean. In the Pacific, Gregg (1998) finds high values of dissipation in the shear zones of the deep jets, but the average dissipation is three to five times lower than our estimates indicate. In the Atlantic, dissipation is also modulated by the shear of the deep jets, but peak dissipation rates are only of modest intensity and about four times less than those shown in Fig. 13 (John Toole 1999, personal communication).

\section{Summary and concluding remarks}

This study was motivated by observations of lowstratification layers in density profiles from the equator in the Indian Ocean at $80.5^{\circ} \mathrm{E}$. Possible mechanisms responsible for the creation of these layers are strain associated with the equatorial deep jets, strain of the equatorial internal wave field, and vertical mixing.

The deep jets were studied based on direct velocity and hydrographic profiles along $80.5^{\circ} \mathrm{E}$. It was found that

- the vertical wavelengths of the zonal deep jets are confined to a narrow band around $650 \mathrm{sm}$ (mode 1517). They have amplitudes of up to $10 \mathrm{~cm} \mathrm{~s}^{-1}$ and contain more than $12 \%$ of the total variance.

- zonal velocities are significantly correlated with vertical displacement with a reversal of phase between $1^{\circ}$ and $2^{\circ}$ off the equator. Zonal velocity leads vertical displacement within $1^{\circ}$, but lags vertical displacement at $2^{\circ}$ off the equator. Furthermore, the zonal flow at $2^{\circ}$ off the equator is opposite in direction to that on the equator.

- the jets show large variability in time. They were strongly developed during January 1991 and July 1993, but were considerably weaker in September 1994. Vertical positions of the jets differ among the cruises and may have reversed between winter and summer.

These observations can be interpreted in terms of free linear equatorial wave theory. The observed phase between vertical displacement and zonal velocity as well as the zonal flow reversal agrees with the meridional structure of a first meridional mode nondispersive Rossby wave.

Previous studies of the equatorial deep jets also showed the dominance of the first meridional mode Rossby wave. Muench et al. (1994) investigated the meridional structure of zonal velocity as well as of potential vorticity in observations from the equatorial $\mathrm{Pa}$ cific. In the western Indian Ocean O'Neill and Luyten (1984) also found a phase reversal of the small verticalscale features between zonal velocities at $1.5^{\circ}$ and $3^{\circ}$ off the equator. Their calculations of drop-lag coherence were significant only for the 720 -sm band at the equator in the data collected over a 1-month period.

The investigation of the internal wave field using finescale data had the following results:

- spectral levels of shear and strain within $1^{\circ}$ of the equator are enhanced by a factor of five compared to levels at $4^{\circ}$ off the equator.

- shear to strain ratio is two throughout the equatorial waveguide.

- with $k_{c}=0.024 \mathrm{cpsm}$, the cutoff wavenumber is 
smallest within $1^{\circ}$ of the equator. For $k_{c} \geq k_{z}>0.25$ cpsm the spectral shape of strain is found to be within $k_{z}^{-1.15} \pm 0.05$ at all latitudes.

Possible energy sources for finescale internal waves are the ocean boundaries, internal instability, and wavewave interaction. Three major ridge systems cross the equator of the Indian Ocean at $64^{\circ}, 72^{\circ}$, and $90^{\circ} \mathrm{E}$. Finescale internal waves generated by the interaction of low vertical mode currents with topography may provide an explanation for the elevated energy levels. Gregg et al. (1995) hypothesized longer timescales for wave-wave interactions to explain the discrepancy between elevated internal wave levels and low dissipation rates. In this case, topographically generated finescale internal waves could have much longer propagation paths as would be expected for midlatitude internal waves. Also, at the surface strong westerly winds prevail at the equator twice a year during the monsoon transition periods. The exerted stress may provide an additional internal wave energy source.

Estimates of the dissipation rate at the equator were performed using the parameterization of Kunze et al. (1990) and finescale data:

- the mean dissipation rate within $1^{\circ}$ of the equator between 500- and 2000-m depth is estimated as $\bar{\epsilon}=7.5$ $\times 10^{-10} \mathrm{~W} \mathrm{~kg}^{-1}$,

- inferred dissipation directly at the equator is more than twice as large,

- low Richardson numbers were found predominately in the layers of low stratification.

Estimates in the other equatorial oceans do not indicate such elevated dissipation levels within the deep jets. This raises the question, whether our estimates are biased to higher values. It is conceivable, for example, that the KWB parameterization does not adequately describe dissipation within the deep jets. John Toole (1999, personal communication) suggested the possibility that Richardson numbers just above $1 / 4$ could persist for many months, without turbulence being generated. Another possibility is that shear measurement noise might contribute to the elevated dissipation values. However, within and below the Pacific equatorial undercurrent, the average dissipation rate correlates well to $\epsilon \sim \overline{S^{3.5}}$, where $S$ is the total shear on a $20-$ m vertical resolution (Peters et al. 1995). The equatorial undercurrent regime and the equatorial abyssal do have some common characteristics, for example, strong background shear and elevated internal wave levels. There is the possibility that a similar dependency between shear and dissipation applies to the deep jets, which would justify the larger dissipation estimates in the equatorial Indian Ocean at $80.5^{\circ} \mathrm{E}$.

Returning to our initial motivation, it is unlikely that the low-stratification layers are actually produced by vertical mixing. The formation timescales for a homogeneous layer solely by vertical mixing is given by
$\tau=\Delta \mathrm{PE} /(\gamma \bar{\epsilon})$, where $\Delta \mathrm{PE}=(1 / 12) N^{2} H^{2}$ is the gain in potential energy per unit mass of a low-stratification layer of vertical extent $\mathrm{H}$ and $\gamma$ the mixing efficiency. Evaluation of $\tau$ for a typical layer of $30 \mathrm{~m}$ and the above inferred dissipation values yields a formation timescale on the order of 30 days. The higher dissipation values in the unstable regions reduce the timescale to a minimum of $5 \mathrm{~h}$. However, the timescales of the instabilities determined from Hazel's 1972 growth rate in Eq. (3) are of about $1 \mathrm{~h}$ only, suggesting that the mixing events are too short to homogenize a larger part of the water column. Furthermore, within the instabilities, the available kinetic energy that needs to be dissipated [Eq. (3)] is on average a factor 10 smaller than $\triangle \mathrm{PE}$ of a $30-\mathrm{m}$ layer.

The distribution of strain by a first meridional mode Rossby wave having the vertical scale of the deep jets showed some correlation with the larger low-stratification layers, but for the smaller layers, no correlation was found. The presence of the deep jets thus leads to larger vertical extents of the layers, but is not responsible for their existence. This result is supported by the fact that elevated strain regions for this Rossby wave are also located at $2^{\circ}$ off the equator, whereas the lowstratification layers are predominately found within $1^{\circ}$. This leaves the finescale internal wave field as the only candidate.

Acknowledgments. We are grateful to John Toole and Eric Firing for their suggestions and comments during the review process and for pointing out errors in the earlier versions of the manuscript. We also thank Eric Kunze and Kurt Polzin for ongoing discussions during data analysis. The CTD data from R/V Franklin cruise $8 / 94$ were kindly made available by M. Tomczak. This study was supported by the Bundesminister für Forschung und Technologie under the German WOCE program, SO73 and SO88, and by the Deutscher Akademischer Austausch Dienst.

\section{REFERENCES}

Böning, C. W., and F. A. Schott, 1993: Deep currents and the eastward salinity tongue in the equatorial Atlantic: Results from an eddyresolving, primitive equation model. J. Geophys. Res., 98, 69916999.

Cairns, J. L., and G. O. Williams, 1976: Internal wave observations from a midwater float. Pt. 2. J. Geophys. Res., 81, 1943-1950.

Desaubies, Y., and M. C. Gregg, 1981: Reversible and irreversible finestructure. J. Phys. Oceanogr., 11, 541-556.

Dewan, E. M., and R. E. Good, 1986: Saturation and the universal spectrum for vertical profiles of horizontal scalar winds in the atmosphere. J. Geophys. Res., 91, 2742-2748.

Eriksen, C. C., 1980: Evidence for a continuous spectrum of equatorial waves in the Indian Ocean. J. Geophys. Res., 85 (C6), 3285-3303

- 1981: Deep currents and their interpretation as equatorial waves in the western Pacific Ocean. J. Phys. Oceanogr., 11, 48-70. , 1982: Geostrophic equatorial deep jets. J. Mar. Res., 40, 143157 . 
— 1985: Some characteristics of internal gravity waves in the equatorial Pacific. J. Geophys. Res., 90, 7243-7255.

Firing, E., 1987: Deep zonal current in the central equatorial Pacific. J. Mar. Res., 45, 791-812.

, 1988: Shallow equatorial jets. J. Geophys. Res., 93 (C8), $9213-$ 9222.

Fofonoff, N. P., 1985: Physical properties of seawater: A new salinity scale and equation of state for seawater. J. Geophys. Res., 90, 3332-3342.

Galbraith, P. S., and D. E. Kelly, 1996: Identifying overturns in CTD profiles. J. Atmos. Oceanic Technol., 13, 688-702.

Gargett, A. E., P. J. Hendricks, T. B. Sanford, T. R. Osborn, and A. J. Williams III, 1981: A composite spectrum of vertical shear in the upper ocean. J. Phys. Oceanogr., 11, 1258-1271.

Garrett, C. J. R., and W. H. Munk, 1975: Space-time scales of internal waves: A progress report. J. Geophys. Res., 80 (3), 291-297.

Gouriou, Y., and J. Toole, 1993: Mean circulation of the upper layers of the western equatorial Pacific Ocean. J. Geophys. Res., 98, 22 495-22 520.

__ B. Bourlès, H. Mercier, and R. Chuchla, 1999: Deep jets in the equatorial Atlantic Ocean. J. Geophys. Res., 104, 21 21721226.

Gregg, M. C., 1976: Temperature and salinity microstructure in the Pacific equatorial undercurrent. J. Geophys. Res., 81, 11801196.

_ 1998: Estimates and geography of diapycnal mixing in the stratified ocean. Physical Processes in Lakes and Oceans, Coastal and Estuarine Studies, Vol. 54, American Geophysical Union, 305-338.

_ J. Geophys. Res., 96 (C9), 16 709-16 719.

- D. P. Winkler, T. B. Sanford, and H. Peters, 1995: Turbulence produced by internal waves in the ocean thermocline at mid and low latitudes. Dyn. Atmos. Oceans, 24, 1-14.

Hayes, S. P., 1975: Preliminary measurements of the time-lagged coherence of vertical temperature profiles. J. Geophys. Res., 80, $307-311$

Hazel, P., 1972: Numerical studies of the stability of inviscid stratified shear flows. J. Fluid Mech., 51, 39-61.

Hines, C. O., 1991: The saturation of gravity waves in the middle atmosphere. Part I: Critique of linear-instability theory. J. Atmos. Sci., 48, 1348-1359.

Hua, B. L., D. W. Moore, and S. Le Gentil, 1997: Inertial nonlinear equilibrium of equatorial flows. J. Fluid Mech., 331, 345-371.

Joyce, T., and C. Corry, 1991: Requirements for WOCE hydrographic programme data reporting. WOCE Tech. Rep. 67/91, 144 pp.

Kawase, M., 1987: Establishment of deep ocean circulation driven by deep-water production. J. Phys. Oceanogr., 17, 2294-2317.

Kunze, E., and T. B. Sanford, 1996: Abyssal mixing: Where it is not. J. Phys. Oceanogr., 26, 2286-2295.

— A. J. Williams III, and M. G. Briscoe, 1990: Observations of shear and vertical stability from a neutral bouyant float. J. Geophys. Res., 95 (C10), 18 127-18 142.

Leaman, K. D., and T. B. Sanford, 1975: Vertical energy propagation of internal waves: A vector spectral analysis of velocity profiles. J. Geophys. Res., 80, 1975-1978.

Luyten, J. R., and J. C. Swallow, 1976: Equatorial undercurrents. Deep-Sea Res., 23, 999-1001.

Moore, D. W., and S. G. H. Philander, 1977: Modeling of the tropical ocean circulation. Marine Modeling, E. D. Goldberg, et al., Eds., The Sea, Vol. 6, John Wiley Interscience, 319-361.

Moum, J. M., T. R. Osborn, and W. R. Crawford, 1986: Pacific equatorial turbulence: Revisited. J. Phys. Oceanogr., 16, 1516-1522.
Muench, J. E., and E. Kunze, 1999: Internal wave interactions with equatorial deep jets. Part I: Momentum-flux divergence. J. Phys. Oceanogr., 29, 1453-1467.

$\longrightarrow$, and - 2000: Internal wave interactions with equatorial deep jets. Part II: Acceleration of the jets. J. Phys. Oceanogr., 30, 2099-2110.

- - , and E. Firing, 1994: The potential vorticity structure of equatorial deep jets. J. Phys. Oceanogr., 24, 418-428.

O'Neill, K., 1984: Equatorial velocity profiles. Part I: Meridional component. J. Phys. Oceanogr., 14, 1829-1841.

— Zonal component. J. Phys. Oceanogr., 14, 1842-1852.

Peters, H., M. C. Gregg, and T. B. Sanford, 1995: On the parameterization of equatorial turbulence: Effect of fine-scale variations below the range of the diurnal cycle. J. Geophys. Res., 100 (C9), 18 333-18 348.

Polzin, K. L., 1996: Statistics of the Richardson number: Mixing models and finestructure. J. Phys. Oceanogr., 26, 1409-1425.

_ J. M. Toole, and R. W. Schmitt, 1995: Finescale parameterizations of turbulent dissipation. J. Phys. Oceanogr., 25, 306328.

Ponte, R. M., 1989: A simple model of deep equatorial zonal currents forced at lateral boundaries. J. Phys. Oceanogr., 19, 1881-1891.

, and J. R. Luyten, 1989: Analysis and interpretation of deep equatorial currents in the central Pacific. J. Phys. Oceanogr., 19, 1025-1038.

, and - , 1990: Deep velocity measurements in the western equatorial Indian Ocean. J. Phys. Oceanogr., 20, 44-52.

,- , and P. L. Richardson, 1990: Equatorial deep jets in the Atlantic Ocean. Deep-Sea Res., 37, 711-713.

Reppin, J., F. Schott, J. Fischer, and D. Quadfasel, 1999: Equatorial currents and transports in the upper central Indian Ocean: Annual cycle and interannual variability. J. Geophys. Res., 104, 15 49515514.

Sanford, T. B., 1991: Spatial structures of thermocline and abyssal waves. Dynamics of Oceanic Internal Gravity Waves: Proc. 'Aha Huliko'a Hawaiian Winter Workshop, University of Hawaii at Manoa, HI, 109-142.

Schott, F., J. Reppin, J. Fischer, and D. Quadfasel, 1994: Currents and transports of the monsoon current south of Sri Lanka. J. Geophys. Res., 99 (C12), 25 127-25 141.

— L. Stramma, and J. Fischer, 1995: The warm water inflow into the western tropical Atlantic boundary regime, spring 1994. J. Geophys. Res., 100, 24 745-24 760.

Send, U., 1994: Accuracy of velocity profile measurements: Effect of tropical and midlatitude internal waves. J. Geophys. Res., 99 (C8), 16 229-16 236.

- M. Visbeck, and G. Krahmann, 1996: Aspects of acoustic transponder surveys and acoustic navigation. IEEE Oceans, 96, $1631-1642$

Spain, P. F., D. L. Dorson, and H. Rossby, 1981: Pegasus: A simple, acoustically tracked velocity profiler. Deep-Sea Res., 28, 15531567.

Thorpe, S. A., 1977: Turbulence and mixing in a Scottish loch. Philos. Trans. Roy. Soc. London, 286, 125-181.

Toole, J. M., and S. P. Hayes, 1984: Finescale velocity-density characteristics and Richardson number statistics of the eastern equatorial Pacific. J. Phys. Oceanogr., 14, 712-726.

Wang, D., D. W. Moore, and L. M. Rothstein, 1994: Exact solutions to Kawase's linear model of deep-ocean circulation. J. Phys. Oceanogr., 24, 2188-2195.

Wunsch, C., 1977: Response of an equatorial ocean to a periodic monsoon. J. Phys. Oceanogr., 7, 497-511. 